\title{
GAS DYNAMICS AND FLOW CHARACTERISTICS OF HIGHLY TURBULENT UNDER- EXPANDED HYDROGEN AND METHANE JETS UNDER VARIOUS NOZZLE PRESSURE RATIOS AND AMBIENT PRESSURES
}

\section{A. Hamzehloo and P.G. Aleiferis ${ }^{*}, 1$}

Department of Mechanical Engineering, University College London, UK

*Author for Correspondence:

Prof. Pavlos Aleiferis

University College London

Department of Mechanical Engineering

Torrington Place, London WC1E 7JE, UK

Tel: $+44-(0) 20-76793862$

E-mail: p.aleiferis@ucl.ac.uk

${ }^{1}$ Currently:

Imperial College London

Department of Mechanical Engineering

Exhibition Road, London SW7 2AZ, UK

Tel: +44-(0)20-75947032

E-mail: p.aleiferis@imperial.ac.uk

Full length article accepted for publication by the International Journal of Hydrogen Energy 


\section{ABSTRACT}

The current study used large eddy simulations to investigate the sonic and mixing characteristics of turbulent under-expanded hydrogen and methane jets with various nozzle pressure ratios issued into various ambient pressures including elevated conditions relevant to applications in direct injection gaseous-fuelled internal combustion engines. Due to the relatively low density of most gaseous fuels such as hydrogen and methane, DI requires high injection pressures to achieve suitable mass flow rates for fast in-cylinder fuel delivery and rapid fuel-air mixing. Such pressures typically form an under-expanded fuel jet past the nozzle exit. Test cases of hydrogen injection with nozzle pressure ratio (NPR) of 10 issued into quiescent air with pressure $P_{\infty} \approx 1,5$ and 10 bar were simulated. Direct comparison between hydrogen and methane jets with NPR=8.5 and $P_{\infty} \approx 1$ was also made. The effect of ambient pressure on features of transient development of the nearnozzle shock structure and tip vortices (vortex ring) was investigated. It was observed that at constant NPR, higher ambient pressure resulted in slightly faster formation of the Mach reflection and shorter Mach disk settlement time. Different mechanisms were observed between hydrogen and methane with regards to transient formation of their initial tip vortex rings. It was found that the initial transient tip vortices of hydrogen jets may also contribute to the flow instabilities at the boundary of the intercepting shock and, unlike for methane, promote fuel-air mixing before the Mach reflection. It was also shown that the nearnozzle shock structure was only affected by NPR regardless of the ambient pressure. Furthermore, no flow recirculation zone was found just downstream of the Mach disk, a finding comparable to all previous experimental investigations. Also, it was observed that a locally richer mixture was created for jets with higher NPR or with higher ambient pressure at constant NPR. Based on the results of the current study, correlations were proposed for the shock cell spacing and jet tip penetration of highly under-expanded jets issued from millimetre-size circular nozzles. 


\section{NOMENCLATURE}

\section{Latin Symbols and Abbreviations}

A

AMG

$B$

AUSM

$C_{A}$

$C_{H}$

$C_{f}$

$C_{p}$

$C_{t}$

$C_{W}$

CFD

CNG

D

$D_{i}$

DI

DNS

$\mathrm{H}_{2}$

$H_{\text {disk }}$

IC

$K$

$K_{m}$

LES

$L_{\mathrm{s}}$

$\dot{M}$

$M a$

$M a_{1}$

$M a_{\text {disk }}$

$M a_{A}$

$M a_{j}$

NPR

$P$

$P_{0}$

$P_{\infty}$

$P_{A}$
Shock Upstream Condition

Algebraic Multigrid

Shock Downstream Condition

Advection Upstream Splitting Method

Coefficient of the New Correlation of the Jet Tip Penetration

Constant of Mach Disk Height Correlation

Coefficient in Jet Penetration Correlation

Specific Heat

Coefficient in Jet Penetration Correlation

Constant of Mach Disk Width Correlation

Computational Fluid Dynamics

Compressed Natural Gas

Nozzle Exit Diameter

Coefficient of Molecular Diffusivity

Direct Injection

Direct Numerical Simulation

Hydrogen

Mach Disk Height

Internal Combustion

Coefficient in Shock Spacing Correlation

Kinematic Momentum Flux

Large Eddy Simulation

Shock Cell Spacing

Momentum Flux

Mach Number

Mach Number at the Nozzle Exit

Mach Number at The Mach Reflection

Shock Upstream Mach Number

Jet Fully Expanded Mach Number

Nozzle Pressure Ratio

Pressure

Upstream (Nozzle) Total Pressure; Injection Pressure

Static Ambient Pressure

Shock Upstream Pressure 


$\begin{array}{ll}P_{B} & \text { Shock Downstream Pressure } \\ \text { PIV } & \text { Particle Image Velocimetry } \\ \text { SGS } & \text { Sub-grid Scale } \\ t & \text { Time After Start of Injection } \\ t_{0} & \text { Nominal Integral Time Scale } \\ T & \text { Temperature } \\ T_{0} & \text { Upstream Total Temperature } \\ T_{\infty} & \text { Ambient Temperature } \\ U_{1} & \text { Nozzle Exit Velocity } \\ U_{A} & \text { Shock Upstream Velocity } \\ W_{\text {disk }} & \text { Mach Disk Width } \\ \text { WALE } & \text { Wall-Adapting Local Eddy-Viscosity } \\ X & \text { Mole Fraction } \\ Y_{\mathrm{c}} & \text { Scalar Mass Fraction } \\ Y_{i} & \text { Mass Fraction of i }{ }^{\text {th }} \text { Species } \\ Z & \text { Axial Distance From The Nozzle Exit } \\ Z_{t i p} & \text { Jet Tip Penetration }\end{array}$

\section{Greek Symbols}

$\begin{array}{ll}\beta & \text { Angle of Inclination in Shock } \\ \Gamma & \text { Scaling Constant } \\ \gamma & \text { Ratio of Specific Heats } \\ \eta_{g} & \text { Taylor Microscale } \\ \eta_{k} & \text { Kolmogorov Length Scale } \\ \eta_{L} & \text { Integral Length Scale } \\ \theta & \text { Shock Deflection Angle } \\ \rho & \text { Density } \\ \rho_{\infty} & \text { Ambient Density } \\ \lambda & \text { Jet Wavelength } \\ \mu & \text { Dynamic Viscosity } \\ \omega & \text { Vorticity magnitude }\end{array}$




\section{INTRODUCTION}

\subsection{Gaseous Fuelling}

One of the proposed solutions to strengthen security of fuel supply and comply with international obligations for reduction of carbon-based emissions is to diversify towards use of more sustainable fuels and cleaner energy sources. More than a few alternative liquid and gaseous fuels have been recommended for sparkignition internal-combustion engines. Gaseous hydrogen $\left(\mathrm{H}_{2}\right)$ has been proposed as, ideally, the most promising carbon-free alternative particularly for road transportation if produced in a sustainable manner. Development of hydrogen-fuelled spark-ignition engines has been investigated experimentally and computationally by various research groups predominantly since the beginning of the past decade [1]. However, the technology of hydrogen-fuelled IC engines has not yet been commercialized due to various technical and political obstacles including: absence of fully developed high-pressure hydrogen injectors with the necessary degree of durability, issues with on-board hydrogen storage and high-pressure fuel delivery systems with suitable crashworthiness characteristics, difficulties in mass production of hydrogen in clean and sustainable ways, the need for significant infrastructural investments for worldwide hydrogen distribution networks, etc. For the past twenty years or so the use of hydrogen has also been widely researched for fuel-cell powered vehicles. However, despite the relatively high efficiency of fuel cells, their manufacturing cost is still expensive and there are also several remaining technical challenges related to their performance under a range of conditions, condensation issues, etc. Therefore, the concept of a hydrogenfuelled combustion engine is still quite appealing for future application on a commercial scale. On the other hand, methane, in the form of compressed natural gas $(\mathrm{CNG})$, has been used on a commercial scale as a relatively cleaner and cheaper alternative fuel for road transportation and power generation [2].

Direct injection (DI) of gaseous fuels into the engine cylinder after intake valve closure [1-5] is believed to be the most preferable fuelling approach for advanced gaseous-fuelled engines. This is because DI can overcome the volumetric efficiency losses that occur with port fuel injection [1-3]. Such losses are associated with the characteristically low densities of gaseous fuels and the long injection duration required that inevitably displaces air during the intake stroke, particularly with hydrogen port fuel injection. In contrast, it has been shown that hydrogen DI can lead to the same, or even higher, volume-specific power than that of conventional gasoline engines [1]. Moreover, the degree of in-cylinder mixture homogeneity or stratification at the time of ignition can be attuned by various DI strategies [3-5]. Typically high pressures are applied for DI fuelling in order to achieve high fuel mass flow rate and consequently rapid fuel-air mixing, particularly with late injection strategies [1,3-5]. High injection pressures result in formation of turbulent under-expanded fuel jets after the nozzle exit [3-5]. Consequently, fundamental understanding of the gas dynamics and sonic/mixing characteristics of under-expanded jets formed upon hydrogen injection from the mm-size holes of typical injectors is indispensable for knowledge and technology transfer that will enable the development of new more efficient high-pressure DI gaseous-fuelled engines. 


\subsection{Under-Expanded Jets}

\subsubsection{Near-Nozzle Shock Structure}

The characteristics of a gaseous jet issuing from a circular nozzle are highly dependent on the ratio of the upstream (nozzle) total pressure $\left(P_{0}\right)$ to the ambient static pressure $\left(P_{\infty}\right)$, specifically the nozzle pressure ratio (NPR). Based on NPR, jets can be characterised as subsonic, moderately under-expanded and highly underexpanded [6-7]. Hydrogen and methane jets with NPR $\geq 4$ are considered to be highly under-expanded. As illustrated schematically in Figure 1 for an under-expanded jet, Prandtl-Meyer expansion fans form at the nozzle lip. Weak compression waves are formed by reflection of the Prandtl-Meyer fans from the jet boundary and form the intercepting shock. The latter shock is ended by a marginally curved strong normal shock, the Mach disk [6-7], which together shape the first shock cell. On a two dimensional plane the intercepting oblique shock and the Mach disk merge at the triple point and produce a reflected shock and a slip line [6-8]. The slip line shows the existence of an annular shear layer within the jet volume. Highly under-expanded jets have two annular shear layers, the inner and outer layer [9-10]. The inner shear layer lies between the high-velocity gas near the jet boundary and the low-velocity jet core, while the outer shear layer (or the mixing layer) lies within the jet boundary and the surrounding medium.

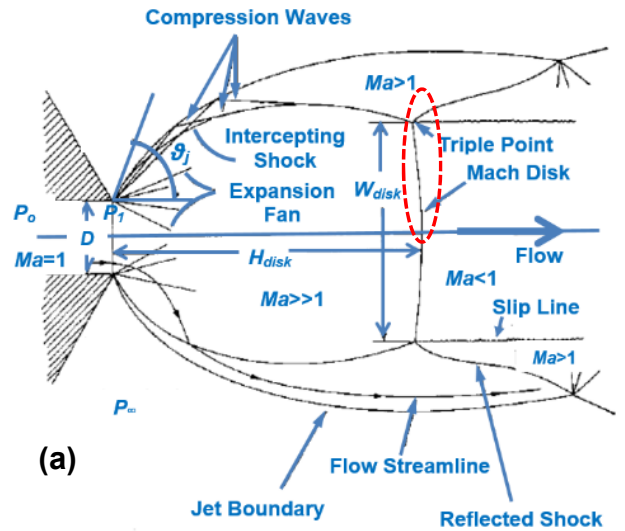

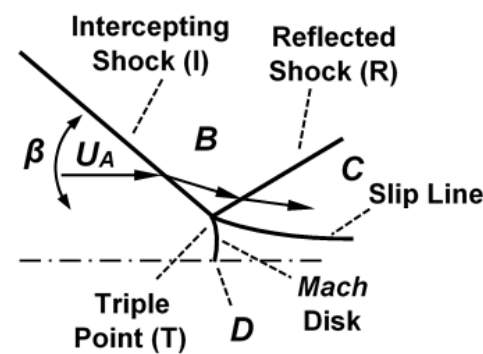

(b)

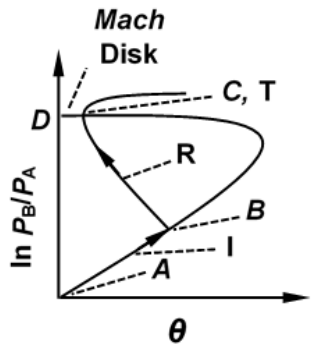

(c)

Figure 1. (a) Schematic of the near-nozzle shock structure of under-expanded jets (based on the visualisation presented by Crist et al. [6]). (b) Schematic of Mach reflection and its corresponding shock locus (detail of dashed circle shown in (a). (c) Diagram of $(\theta, P)$ based on visualisation presented by Hornung [8] and in reference to points $A, B, C$ and $D$ shown in (b).

The cause of the Mach disk formation and generally the complex near-nozzle shock structure can be explained by solving the gas dynamics conservation equations deriving 'shock-jump relations' that relay the downstream conditions in an oblique shock to its upstream. According to this, there are two major reflections that may occur in all types of flows (steady, quasi-steady and unsteady), regular and Mach reflections [8]. Figure 1 also includes a schematic of the Mach reflection process. If the angle of inclination $\beta$ passes a critical value (that is a function of the upstream Mach number and the ratio of specific heats) then the regular reflected shock cannot correct the flow direction downstream of the intercepting oblique shock and its locus does not reach the pressure axis [8]. Therefore, a near-normal shock forms which allows the triple point to move away from the wall or the symmetry plane. This process is called Mach reflection. Similarly to the flow upstream, the flow after the Mach reflection is parallel to the wall (or a symmetry plane). The Mach disk in highly under-expanded jets is a Mach reflection from the nozzle symmetry plane. A vortex sheet (slip 
line) separates the flow stream processed by the reflected and near-normal shocks (which are supersonic and subsonic, respectively). Density, velocity and entropy are discontinuous across the slip line but pressure and streamline deflection must be continuous [8]. This may trigger a turbulent mixing process within the jet core [10]. For relatively high levels of under-expansion, e.g. NPR $\geq 8$, the subsonic core downstream of the Mach disk accelerates quickly and becomes supersonic again. This then gives rise to a second shock cell that may be similar to the first shock cell and contains a normal shock similar to the Mach reflection [7].

\subsubsection{Mach Disk Dimensions}

The dimensions of the Mach disk in under-expanded jets, i.e. the distance between the disk and the nozzle exit (on the nozzle axis), termed the Mach disk height $H_{\text {disk }}$, and the distance of the two triple points, termed the Mach disk width $W_{d i s k}$, have both been the subject of several experimental and computational studies over the past 50 years, mainly for aeronautical applications. The primary importance of these parameters comes from the fact that they can provide significant information regarding the location and size of the annular shear layers and hence mixing characteristics of this type of flow. These parameters may also be used for direct comparison of various under-expanded jets and also for validation of their computational predictions. In an early attempt Crist et al. [6] used a hot-shot wind-tunnel facility and Schlieren to study the near-nozzle shock structure and to measure the $H_{\text {disk }}$ for a variety of gases including Nitrogen, Argon, Helium and $\mathrm{CO}_{2}$. They concluded that the relation between NPR and Mach disk height could be described by:

$$
\frac{H_{d i s k}}{D}=C_{H} \sqrt{\frac{P_{0}}{P_{\infty}}}
$$

Several other researchers attempted to estimate the constant of Equation (1). For instance, Ashkenas and Sherman [11], Velikorodny and Kudriakov [12] and Vuorinen et al. [13] suggested $C_{H}$ values of 0.67, 0.63 and 0.62 , respectively. The authors of the current work found that for hydrogen jets with values of NPR up to $10, C_{H}$ had a value of $\sim 0.65$ [14]. An average value of $C_{H} \approx 0.71$ has been measured for hydrogen jets with NPR in the range 8.5-70 [14]. An empirical correlation comparable to Equation (1) can also be used to estimate the Mach disk width with a respective constant $C_{W}$. Various attempts have also been made to obtain a generalised value of $C_{W}$ for under expanded jets [12-13], however, because of the noticeably greater nonlinearity between $W_{\text {disk }}$ and NPR in comparison to that of $H_{\text {disk }}$ and NPR, there is still not a unique correlation or, at least, a widely accepted value of $C_{W}[13]$.

\subsubsection{Core Shock Cells}

The spacing length of the core shock cells is another important parameter in under-expanded jets. Depending on NPR, a number of shock cells may form within the jet core to allow the static pressure inside the jet to decrease gradually to that of the surrounding ambient. The shock cells are formed by reflection of radially propagating oblique shocks and expansion fans from the jet boundary. The reflection process may be repeated several times until the associated shocks are dissipated by the highly turbulent mixing region (typically by merging of the inner and outer shear layers). These frequent reflections have a quasi-periodic 
nature [15-17]. Studies of the near-nozzle shock structures have been done primarily to investigate the aeroacoustics involved in the physics of under-expanded jets, especially the level of sound emitted (screech tone) from the expansion process due to the quasi-periodic nature of the shock cells $[16,18]$. Similarly to the dimensions of the Mach disk, the spacing of the shock cells is also useful for comparison and model validation purposes.

Emden [19] carried out the first quantitative experimental study of the near-nozzle shock cell structures using schlieren visualisation. He noticed that the jets had a periodic structure with a specific wavelength. Using various nozzle designs, he discovered that the wavelength $\lambda$ could be expressed as a function of the nozzle exit diameter $D$ and NPR:

$$
\lambda=K D \sqrt{N P R-1.9}
$$

The shock cell spacing $L_{\mathrm{s}}$ was found to be the longest wavelength. This resulted in $K$ values in the range of 0.77-1.02 depending on nozzle type, with a mean value of 0.88. Prandtl [20] studied theoretically the shock cell spacing by a linear vortex sheet jet model and by developing an approximate solution. He considered slight perturbations about a steady state and estimated a mean value of $K$ of 1.2. This was too high in comparison to Emden's one [19]. Finally, after attempts by various researchers, a complete vortex-sheet shock-cell solution for moderately under-expanded jets was derived by Pack [18]:

$$
L_{\mathrm{s}}=K D \sqrt{M a_{j}^{2}-1}
$$

where $K$ is in the range of $1.15-1.3$ and $M a_{j}$ is the fully expanded jet Mach number. By assuming $K=1.22$, $\gamma=1.41$ and using Bernoulli's equation to replace the Mach number by NPR in Equation (3), Pack [18] derived the following correlation for shock cell spacing:

$$
L_{\mathrm{s}}=2.695 D \sqrt{\mathrm{NPR}^{0.291}-1.205}
$$

The vortex sheet shock-cell model used by Pack [18] is not valid apart from where the mixing layer is thin, i.e. near the nozzle exit. Tam and Tana [21] and Tam et al. [22] developed and extended a linear shock-cell solution for jets with a realistic mean flow profile using the method of 'multiple-scales expansion'. They concluded that a good approximation to the spacing of the shock cell structure could be given by:

$$
L_{\mathrm{s}}=\frac{\pi D \sqrt{\left(M a_{j}^{2}-1\right)}}{2.405}
$$

For under-expanded jets of relatively low Reynolds number, as well as for micro jets, smaller cell spacing values than those derived by Equation (5) have been reported in the literature [23-25]. Thicker boundary layers due to low Reynolds numbers and associated viscous effects were suggested as possible causes for this discrepancy. For instance, using schlieren imaging of under-expanded jets issued from micro nozzles with diameters in the range 100-1000 $\mu \mathrm{m}$, Phalnikar et al. [25] suggested an empirical correlation for shock cell spacing as follows: 


$$
L_{\mathrm{s}}=D\left(0.57 M a_{j}^{2}-0.15\right)
$$

This leads to about $10-30 \%$ difference in $L_{\mathrm{s}}$ when compared to Equation (5).

\subsubsection{Jet Penetration}

The jet's tip penetration $Z_{t i p}$ is the key parameter of under-expanded fuel jets when it comes to mixture formation in engines. The jet penetration under conditions similar to those prevailing in DI gaseous-fuelled engines (under-expanded with Reynolds number of the order $10^{5}-10^{6}$ ) has been found to obey a linear dependency on the square root of time [26-28]. Turner [29] developed a model to approximate the geometry of a gaseous jet as a spherical head vortex and a quasi-steady jet region that conveys the momentum. Hill and Ouellette [30] employed this model to develop an analytical relationship for the tip penetration of underexpanded jets. Specifically, it was assumed that momentum is constantly supplied through the nozzle orifice and is passed between the quasi-steady region and the head vortex. Hill and Ouellette [30] used formulations derived by Ricou and Spalding [31] for the entrainment of low momentum ambient air and suggested a correlation for the jet tip penetration $Z_{t i p}$ as:

$$
\mathrm{Z}_{\text {tip }}=\Gamma\left(\frac{\dot{M}}{\rho_{\infty}}\right)^{0.25} t^{0.5}
$$

where $\dot{M}$ is the momentum flux supplied by the nozzle and $\Gamma$ is a scaling constant which is a function of the entrainment coefficient and the ratio of the jet's head vortex diameter to its tip penetration [28]. The value of $\Gamma$ for turbulent under-expanded jets issued from round nozzles is about 3 [27].

By assuming self-similar velocity profile and employing conservation of momentum, Abraham [32] used an expression for centreline velocity of turbulent jets developed originally by Schlichting [33] in order to derive an expression for the jet tip penetration $\left(\mathrm{Z}_{t i p}\right)$ as follows:

$$
\mathrm{Z}_{\text {tip }}=\sqrt{\frac{3 C_{f}}{4 \pi C_{t}}\left(K_{m} \frac{\rho_{1}}{\rho_{\infty}}\right)^{0.5} t}
$$

where $K_{m}=A_{1} U_{1}^{2}$ is the kinematic momentum flux of the round jet at the nozzle exit, $C_{f}$ is a certain fraction of the local steady centreline velocity and $C_{t}$ is a constant that relates the jet diffusivity to the jet kinematic momentum [32-33]. Different combinations of $C_{f}$ and $C_{t}$ have been suggested in the literature. For example, Song and Abraham [34] employed $C_{t}=0.0113$ and $C_{f}=0.305$ and successfully managed to reproduce the penetration curve of a round jet.

\subsection{Present Contribution}

The near-nozzle shock structure and mixing characteristics of under-expanded jets have been subject to various experimental investigations since the mid-1950s, using predominantly air or nitrogen issued from relatively large aerospace-type nozzles. Recently large eddy simulation (LES) was employed to investigate under-expanded methane and nitrogen jets $[13,35]$. However, very limited experimental and computational 
studies are available in the literature on under-expanded hydrogen and methane jets and specifically with respect to applications in DI gaseous engines [36-41]. Recently, the authors of the present paper used LES to investigate the near-nozzle shock structure and mixing characteristics of highly under-expanded hydrogen and methane jets with $\mathrm{NPR}=8.5-70$ issuing from a circular nozzle into a low ambient pressure $\left(P_{\infty} \approx 1\right.$ bar $)$ $[14,42]$. The present computational work aims to extend the previous studies on under-expanded hydrogen and methane jets by investigating effects of ambient pressure on the key characteristics of this type of flow. To the best of the authors' knowledge the current study is the first to employ LES in order to study sonic and mixing characteristics of under-expanded hydrogen and methane fuel jets under various NPR values and ambient pressures (including elevated engine-like ambient pressures). The main objectives of the current work can be summarized as follows:

- Investigate the transient formation features of the Mach disk and the near-nozzle shock structures with hydrogen and methane fuels in relation to NPR.

- Conduct quantitative and comparative studies on the initial transient tip vortices (initial vortex ring) and their effect on the mixing characteristics and shear layer formation in hydrogen and methane underexpanded jets.

- Study the effect of elevated ambient pressure under constant NPR on the near-nozzle and mixing characteristics of hydrogen jets.

- Formulate empirical correlations for shock cell spacing and jet tip penetration of under-expanded hydrogen and methane jets.

\section{METHODOLOGY}

\subsection{Numerical Approach}

The numerical approach employed in order to conduct LES studies has been described in preceding publications by the current authors [14, 42]. Nevertheless, for the immediate benefit of the reader, a brief description is also included here.

Preconditioned compressible formulation of the governing equations of viscous flows (mass, momentum and energy conservations), as well as a species transport equation, were solved using the finite-volume methodology within the STAR-CCM $+{ }^{\circledR}$ code [43-45]. A second-order implicit time marching method was combined with a central differencing scheme for convective fluxes in order to discretize the aforementioned partial differential governing equations and produce a linearized system of equations [44]. The matrix of the linearized system of equations was then solved simultaneously (coupled approach [45]) and by using the Gauss-Seidel iterative technique in conjunction with an algebraic multigrid (AMG) method [43-45]. A modified version of the advection upstream splitting method ( $\mathrm{AUSM}^{+}$-up) [46] was applied in order to express the inviscid fluxes. $\mathrm{AUSM}^{+}$-up is accurate and robust in solving fluid flows containing arbitrary range of velocity magnitude and particularly high speed flows that contain extreme discontinuities such as shock waves [46]. Since direct numerical simulation (DNS) is not feasible for the complicated high-speed 
flow that is investigated in this work, LES was employed instead. For LES the governing equations are filtered so that turbulence scales greater than a filter size (typically associated with the grid resolution) are resolved directly and the effect of scales smaller than that are accounted for by means of sub-grid scale (SGS) modelling [47]. In the present study the wall-adapting local-eddy viscosity (WALE) SGS model which is based on the square of the velocity gradient tensor (can take into account both shear and rotation) was applied [48]. In comparison to the classical Smagorinsky model [47], the WALE model produces zero eddy viscosity in case of a pure shear (may occur in free jets) that makes it capable of reproducing turbulent transitional processes more accurately through the growth of unstable modes [48].

The molecular mixing rate of the species was taken into account using the Chapman-Enskog theory for gaseous diffusion [49]. Specifically, a coefficient of molecular diffusivity $D_{\mathrm{i}}$ was defined as the binary diffusivity of an air-hydrogen or air-methane system [14, 49]. The coefficient of molecular diffusivity $D_{i}$ was calculated to be $7.942 \cdot 10^{-5}, 1.589 \cdot 10^{-5}$ and $7.942 \cdot 10^{-6} \mathrm{~m}^{2} / \mathrm{s}$ for hydrogen injection with $P_{\infty}=1,5$ and 10 bar ambient pressure, respectively. For methane, $D_{i}$ was calculated to be $2.1 \cdot 10^{-5}$. The dynamic viscosity was obtained using Sutherland's law [14].

It is also noted that in addition to the present computational framework the current authors also developed an open-source solver with fourth-order accurate temporal discretization accuracy and with the KNP central flux splitting scheme using OpenFOAM ${ }^{\circledR}$ libraries [50], specifically for modelling under-expanded jets [51]. A basic comparison between the results of the new open-source solver and the STAR-CCM $+{ }^{\circledR}$ framework is also provided and discussed briefly in the current paper as means of further validation of the commercial code. However, due to the very high velocity at the nozzle exit of the under-expanded hydrogen jets, LES modelling with the OpenFOAM ${ }^{\circledR}$ solver required a considerably low constant time step (in the order of nanosecond) to maintain high accuracy and stability, hence within the objectives of the current study it was decided to concentrate on the results obtained with STAR-CCM $+{ }^{\circledR}$.

\subsection{Simulation Setup and Validation}

The computational setup and its preliminary validation process have been discussed in earlier published studies by the current authors [14, 42]. However, a brief description of the setup and validation are also included here for completeness with some additional discussion.

\subsubsection{Computational Setup}

Figure 2 presents the computational domain. This consisted of a high pressure tank that was linked to a low pressure air-containing chamber by a converging nozzle with exit diameter $D=1.5 \mathrm{~mm}$. The geometry and dimensions of the converging nozzle were obtained from an experimental test case available in the literature [40] to enable validation work. The current work examined under-expanded hydrogen $(\mathrm{NPR}=8.5,10)$ and methane $(\mathrm{NPR}=8.5)$ jets issued into quiescent air ambient with various pressures. The pressure of the ambient was kept constant at $P_{\infty}=98.37 \mathrm{kPa}$ for the cases with NPR=8.5. For hydrogen jets with NPR=10, in addition to $P_{\infty}=98.37 \mathrm{kPa}$, two additional elevated ambient pressures, $P_{\infty}=491.85$ and $983.70 \mathrm{kPa}$ were also 
investigated. For convenience, within the rest of this paper, cases with ambient pressures of $P_{\infty}=98.37$, 491.85 and $983.70 \mathrm{kPa}$ are referred to as cases with $\sim 1, \sim 5$ and $\sim 10$ bar ambient pressure, respectively. For all simulations, an initial constant temperature of $295.4 \mathrm{~K}$ and $296 \mathrm{~K}$ was employed for the fuel and air containing volumes, respectively. Following practices in the literature [13, 35, 37] and also based on the discussion provided in [14], adiabatic slip boundary conditions were assigned to the nozzle and high-pressure tank boundaries.
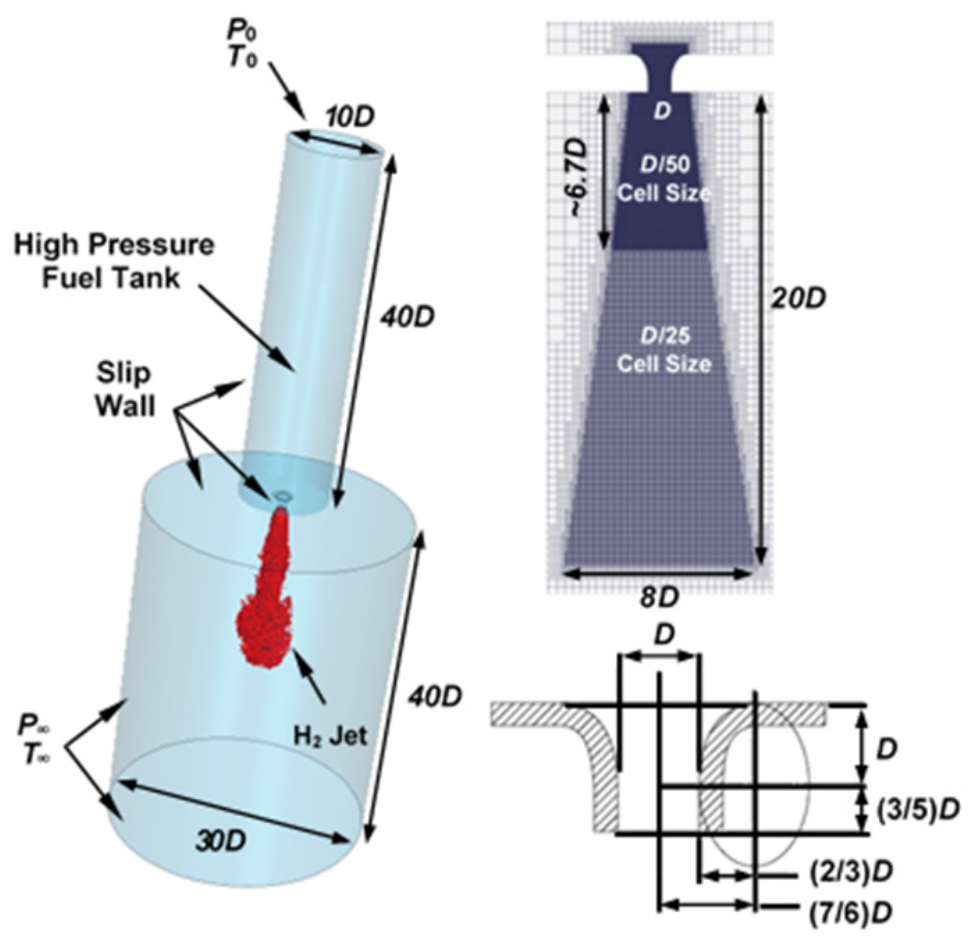

Figure 2. Left: The domain configuration and dimensions. Right-top: Zoomed view of the refined areas. Rightbottom: Nozzle profile and its dimensions based as provided by Ruggles and Ekoto [40].

A computational mesh with hexahedral elements was created within STAR-CCM $+^{\circledR}$. The meshing process produced cubic finite volume elements with identical size in all dimensions as shown in Figure 2. This resulted in a grid without cell stretching which typically provides enhanced numerical accuracy and also avoids the singularity linked to polar grids. In order to capture the flow details inside the nozzle, the shock structure very close to the nozzle exit and the mixing process downstream of the nozzle, a conical refined area was implemented inside the computational domain. This covered the nozzle volume and a length of $20 D$ downstream of the nozzle exit. The refinement inside the nozzle volume and within a distance of $\sim 6.7 D$ downstream the nozzle exit had a cell size of $\sim 0.03 \mathrm{~mm}(D / 50)$, whilst further downstream it had a cell size of $\sim 0.06 \mathrm{~mm}$. By employing a four-level grid coarsening, the spatial resolution expanded to its largest size of $1.0 \mathrm{~mm}$ close to the boundaries of the low pressure ambient. In total $\sim 13.5 \mathrm{M}$ hexahedral cells filled the computational domain. According to previous investigations [13, 35, 37] the spatial resolution employed in the present study was considered fine enough to capture the shock waves embedded in under-expanded jets and also to resolve adequately the level of turbulent energy of this kind of flow within reasonable computational power. A comparison of the spatial resolution of the current study with key scales of turbulent motion, i.e. integral $\left(\eta_{L}\right)$, Taylor $\left(\eta_{g}\right)$ and Kolmogorov $\left(\eta_{k}\right)$, can provide some perspective of the explicitly 
resolved scales [47]. A recent experimental study of under-expanded jets by particle image velocimetry (PIV) [52] quantified integral length scales in the range $\eta_{L}=0.1-0.4 D$ for distances downstream of the nozzle exit in the range $2 D-12 D$. Using typical estimate processes for Taylor and Kolmogorov scaling on the basis of $\eta_{L}$, turbulent kinetic energy, dissipation rate and kinematic viscosity (e.g. see [47]), under conditions of hydrogen jetting with NPR=10 $\left(P_{\infty}=1\right.$ bar, $\left.U_{1} \approx 1300 \mathrm{~m} / \mathrm{s}\right)$, led to values of $\eta_{k}$ of the order $2 \cdot 10^{-6} \mathrm{~m}$ and $\eta_{g}$ of the order $4 \cdot 10^{-5} \mathrm{~m}$. The grid resolution of $D / 50$ and $D / 25$ over the two main refinement volumes of the current study was $3.0 \cdot 10^{-5}$ and $6.0 \cdot 10^{-5} \mathrm{~m}$, respectively. This means that the current LES framework was nominally able to resolve eddies down to about Taylor size. Further spatial refinement to resolve scales close to Kolmogorov would require a spatial resolution finer by more than one order of magnitude which would make running times and analysis impractical over typically available computational resources.

Since modelling transient compressible in-nozzle flows requires very small time steps (usually of the order of nanoseconds and mainly because of the existence of high density gradient inside the nozzle volume) the common practices in the literature is to omit it by applying an arbitrary pressure gradient inside the nozzle $[13,37]$. However, in the present LES study, the in-nozzle transient process was taken specifically into the account. The simulations started from a rest condition where the entire high-pressure tank and a very small part of the converging nozzle volume (up to $\sim 1.4 D$ upstream the nozzle exit) were filled by the gaseous fuel. Quiescent air occupied the low-pressure chamber and the remaining section of the nozzle volume. The nominal integral time scale $t_{0}$ of an under-expanded gaseous jet was calculated to be $\sim 6.2 \cdot 10^{-7} \mathrm{~s}$ and $\sim 1.8 \cdot 10^{-6}$ $\mathrm{s}$ for under-expanded hydrogen and methane jets, respectively [13-14]. A time-step in the range of $1 \cdot 10^{-9}-$ $5 \cdot 10^{-9} \mathrm{~s}$ was used at the early stages of the simulation (for modelling the in-nozzle transient flow); this was then gradually increased to a value in the range $2 \cdot 10^{-8}-5 \cdot 10^{-8} \mathrm{~s}$ depending on the injection pressure. For the cases with elevated ambient pressure (at constant NPR), it was noticed that a lower time-step had to be used in order to maintain stability of the solution. This was attributed to the existence of higher level of momentum gradients inside the nozzle. The nominal integral time scale of the flow was, in any case, 10 times larger than the aforementioned time steps; this made them satisfactory for capturing the turbulent temporal fluctuations at the expense of practical computational cost.

\subsubsection{Model Validation}

As discussed comprehensively in $[14,42]$ in order to validate the current computational framework first a simulation of hydrogen injection with $\mathrm{NPR}=10\left(P_{\infty} \approx 1\right.$ bar $)$ was setup based on the experimental work of [40]. Mach disk dimensions (its height and width), as well as the reflected shock angle, were predicted by LES very close to values that could be extracted from the Schlieren images of [40]. The present LES investigation predicted higher values for the Mach disk height and width by $1.3 \%$ and $3.0 \%$, respectively (3.09 $\mathrm{mm}$ and $1.34 \mathrm{~mm}$ ) compared to those stated in [40] (3.05 $\mathrm{mm}$ and $1.30 \mathrm{~mm}$, respectively). In further agreement with [40], the present LES study predicted the reflected shock (at the triple point) to be inclined $\sim 28^{\circ}$ against the nozzle centreline axis. The reflected shock angle for the methane test case (NPR=8.5) was measured to be $\sim 28.5^{\circ}$, which in addition to the Mach disk height $\left(H_{\text {disk }} \approx 1.9 D\right)$ were in agreement with the 
recent work of Vuorinen et al. [13, 37] on a nozzle of $1.4 \mathrm{~mm}$ dimeter, NPR=8.5 and under-expanded nitrogen and methane jets. Similarly to the experimental observations of [40], it was also seen that hydrogen and air were mixing outside of the intercepting shock, before the Mach reflection. This means that not all of the incoming hydrogen passed through the Mach disk surface. For methane, similarly to [37], mixing did not occur before the Mach reflection. The mixing process at the boundary of the intercepting shock in underexpanded jets is discussed further later in the current paper.

In $[14,42]$ it was shown that jet tip penetration curves of the hydrogen and methane under-expanded jets issued into $P_{\infty}=1$ bar collapsed onto a single trend line by applying an empirical relationship proposed by Ouellette and Hill [27] (similarly to the under-expanded nitrogen jet modelled by Vuorinen et al. [13]). Figure 3 shows that the penetration curve of the hydrogen jets issued into the elevated ambient pressures of $P_{\infty}=5$ bar and 10 bar also collapsed onto a single line using the relationship of [27].

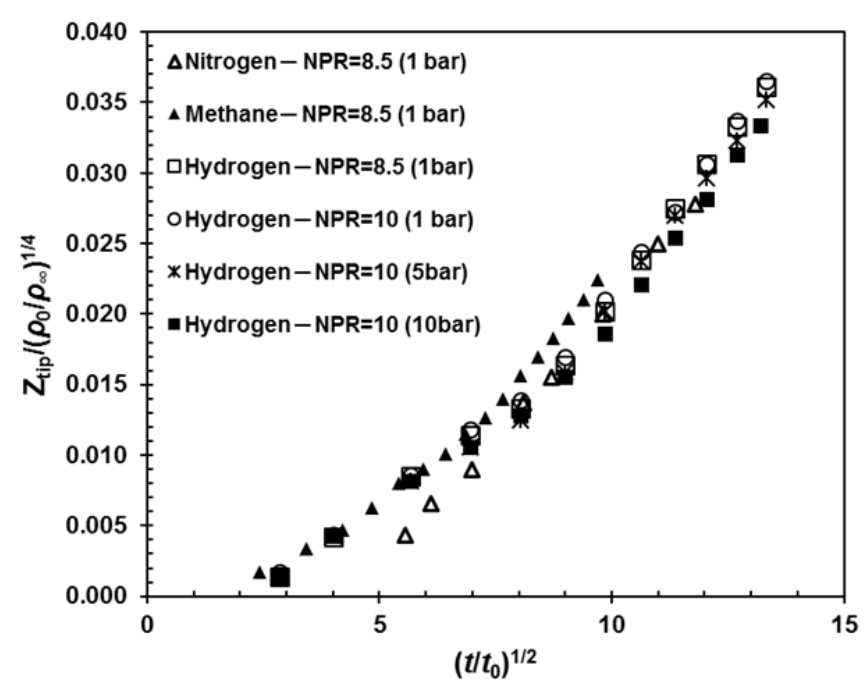

Figure 3. Variation of a modified jet tip penetration (based on relationship of [27]) versus a normalized time parameter. Comparison between hydrogen and methane jets studied in the current LES study with a nitrogen jet available in the literature [13].

\section{RESULTS AND DISCUSSION}

\subsection{Transient Shock Dynamics and Jet Development}

The early stages of near-nozzle shock development and Mach disk formation is presented in Figure $\mathbf{4}$ for the hydrogen injection case with NPR $=10$ issued into air ambient at $P_{\infty} \approx 1$ and 5 bar. Although the transient stages were slightly different, for both cases the Prandtl-Meyer expansion fans formed at the nozzle lip just after hydrogen's entrance into the domain. At the beginning of the simulation process, a subsonic jet started issuing from the nozzle exit and soon after it turned into a moderately under-expanded jet with $M a_{1} \approx 1$ (at $t \approx 8$ $\mu$ for the case with $P_{\infty} \approx 1$ bar). At this condition the expansion fans reflected from the jet boundary and formed the compression waves (fans) which consequently shaped the curvy intercepting shocks. The initial shock cell formed by the intercepting shocks and the intersecting expansion and compression fans (see the 8 $\mu$ snapshots of Figure 4). The in-nozzle flow exhibited a noticeable transient behaviour and when the nozzle exit pressure passed the threshold of $P_{1} / P_{\infty} \geq 2$ at $t \approx 12-13 \mu$ s (for $P_{\infty} \approx 1$ bar) the jet became highly 
under-expanded with a tiny Mach disk. This means that the intersecting fans of the first cell were not 'strong enough' to correct the flow direction and, as discussed earlier, the existence of a Mach reflection was indispensable. The width of the Mach disk then grew gradually until it reached quasi-steady dimensions. This is attributed to the gradually increasing upstream Mach number during the initial transient process. In fact, increasing $M a_{1}$ would increase the overall deflection angle of the oblique shocks of the first cell, therefore a larger portion of the flow should pass through a Mach reflection, i.e. a wider Mach disk would be formed [8]. The fluctuating nature of the Mach disk dimensions (before its settlement at a final semi-steady location), has been attributed to the fluctuating behaviour of the upstream flow of the Mach reflection [14, 53].

Comparison between jets with different ambient pressures in Figure 4 shows that higher ambient pressure resulted in faster formation of the Mach reflection (Mach disk). A difference of 2-3 $\mu$ s was observed in the formation of the Mach disk between the hydrogen jets presented in Figure 4. It is worth mentioning that the transient process of the near-nozzle shock development for the hydrogen jet with NPR $=10$ issued into the ambient with $P_{\infty} \approx 10$ bar (not shown here) revealed a similar behaviour to the jet with $P_{\infty} \approx 5$ bar but exhibited a Mach reflection $\sim 1 \mu$ s earlier. Therefore, it can be concluded that higher ambient pressure under a fixed value of NPR may result in faster near-nozzle shock development and shorter Mach disk settlement time. Shorter temporal and spatial fluctuations around the final steady values were found for the Mach disk dimensions by increasing the ambient pressure under fixed NPR. The difference between the initial transient shock formation in jets with similar NPR but different $P_{\infty}$ is due to the difference in the aforementioned innozzle transient process [14]. A relatively higher in-nozzle momentum and density would result in shorter transient conversion from subsonic to sonic/supersonic flow at the nozzle exit that would advance the formation of the Mach reflection.

The hydrogen jet with NPR=10 and $P_{\infty} \approx 1$ bar in Figure 4 showed similar behaviour to the hydrogen jet of $\mathrm{NPR}=8.5$ with $P_{\infty} \approx 1$ bar in [14]. However, it is noted that the methane jet with NPR=8.5 and $P_{\infty} \approx 1$ bar exhibited slightly different behaviour. Widely spaced initial intercepting shocks were noticed that did not get close during the transient process and formed a wide Mach disk from the very beginning of the injection process. Moreover, it was observed that for the hydrogen jet with NPR=30 and $P_{\infty} \approx 1$ bar the transient shock development progressed similarly to that of the methane jet with NPR=8.5 ( $P_{\infty} \approx 1$ bar $)$, i.e. with the formation of a wide Mach disk from the very beginning [14]. At a constant inclination angle, jets with higher $M a_{A}$ or lower ratio of specific heats $(\gamma)$ would produce more flow deflection which means that formation of a wider Mach reflection is expectable [8]. Methane has a lower $\gamma$ than hydrogen and the hydrogen jet with NPR=30 had relatively higher $M a_{A}$ [14]. The transient evolution of the Mach disk dimensions and the near-nozzle shock structure of both methane and hydrogen jets are comparable with the recently published schlieren observations of Rogers et al. [54]. 

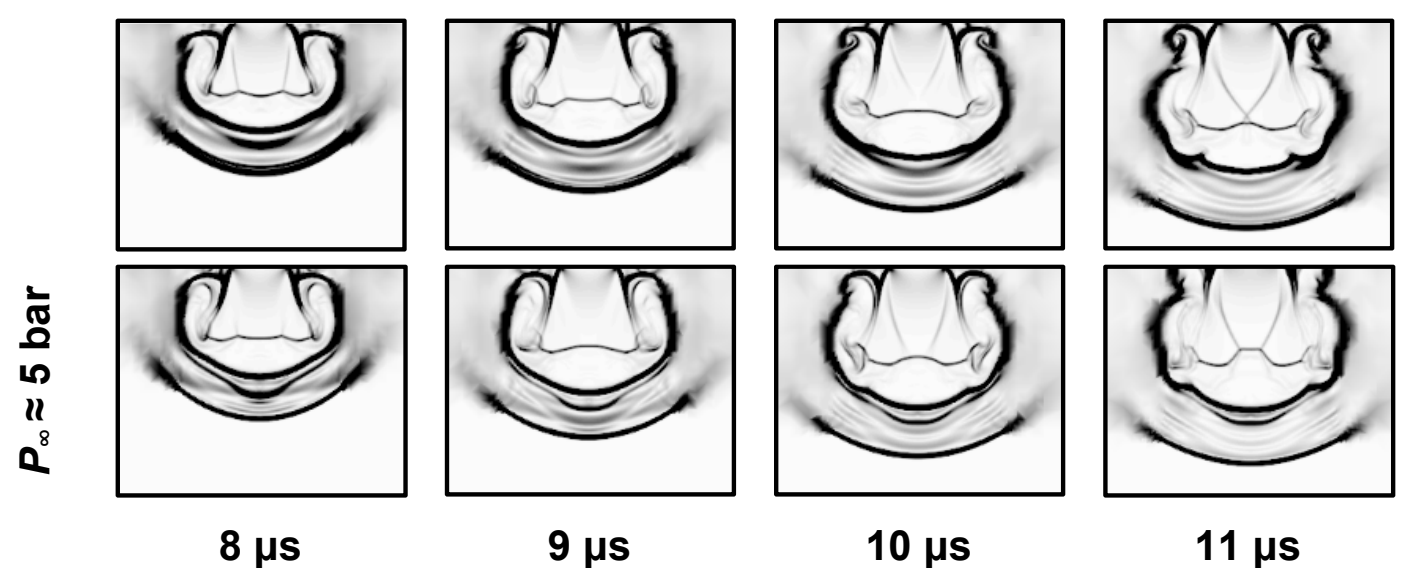

$9 \mu \mathrm{s}$

$10 \mu \mathrm{s}$

$11 \mu \mathrm{s}$
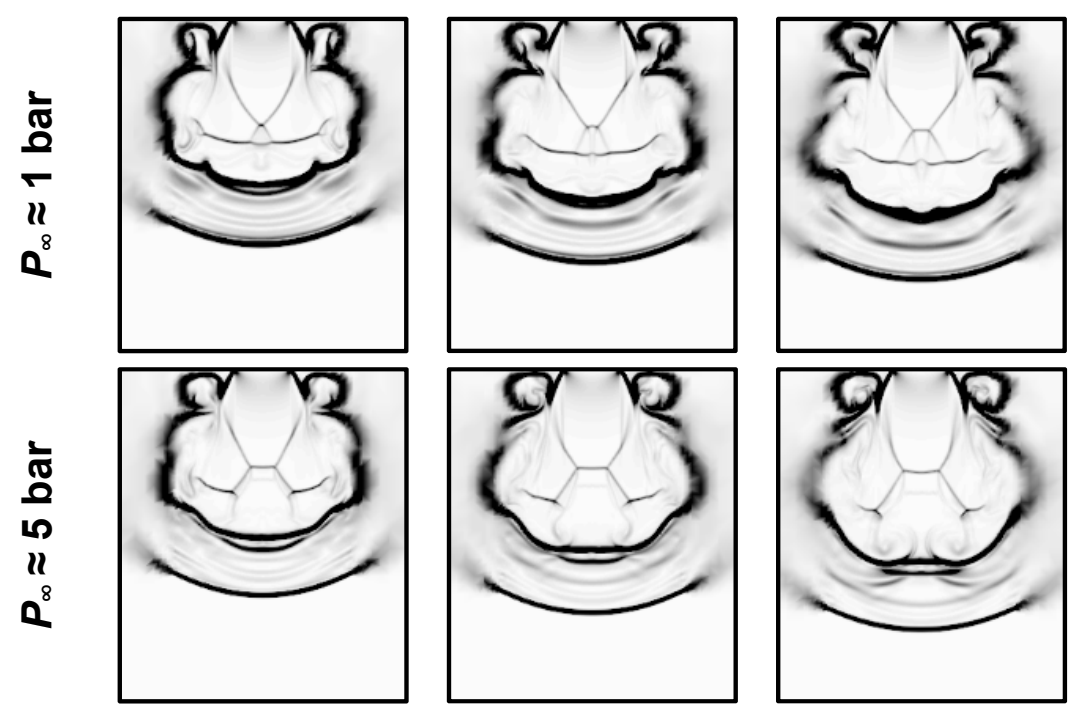

$12 \mu \mathrm{s}$

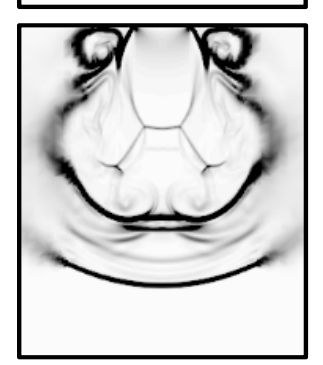

$13 \mu \mathrm{s}$

$14 \mu \mathrm{s}$
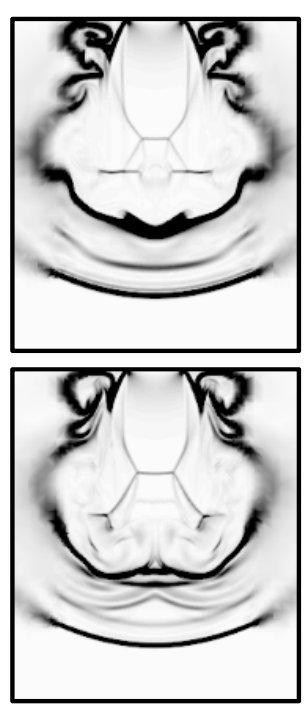

$15 \mu \mathrm{s}$
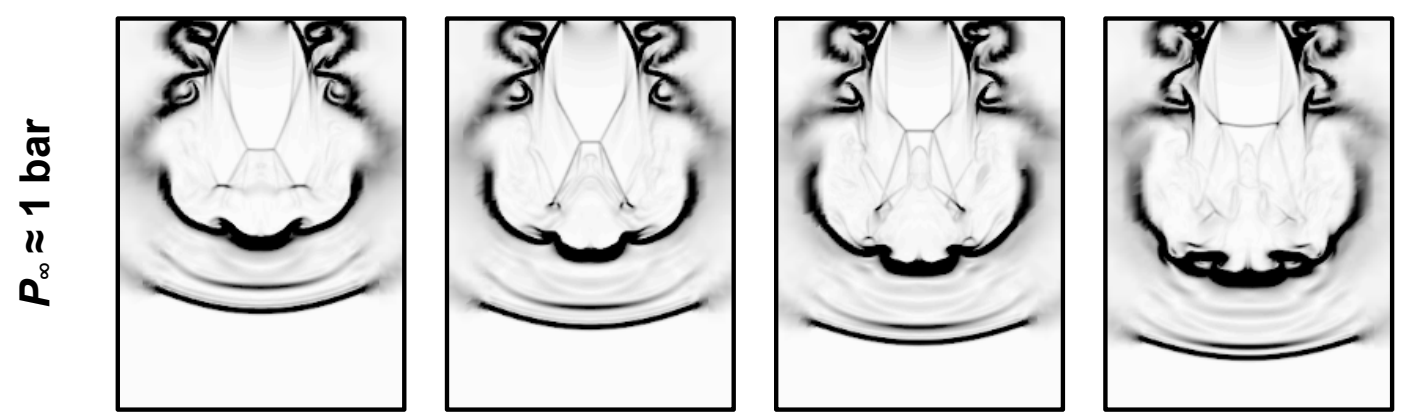

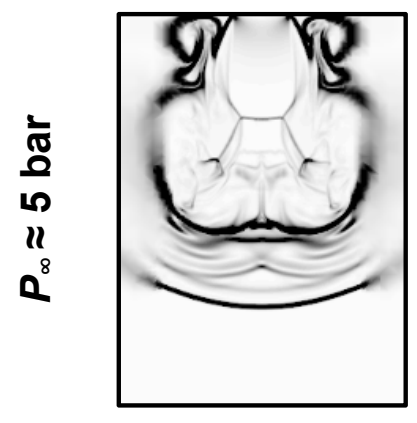

$16 \mu \mathrm{s}$

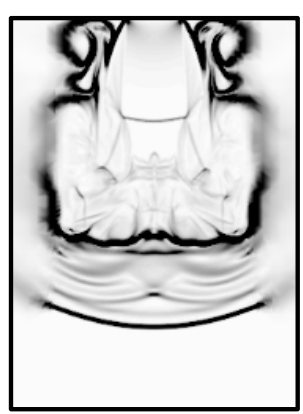

$17 \mu \mathrm{s}$

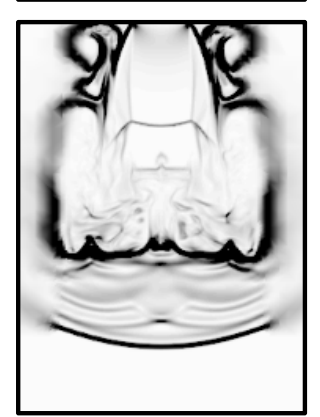

$18 \mu \mathrm{s}$

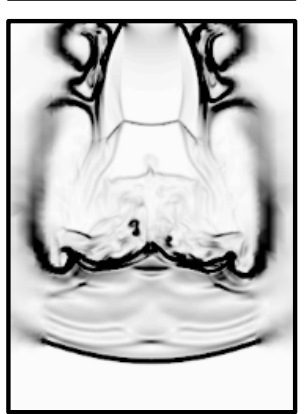

$19 \mu \mathrm{s}$

Figure 4. LES prediction of transient development of the near nozzle shock structure and Mach disk in underexpanded hydrogen jets with $N P R=10$ and $P_{\infty} \approx 1$ and 5 bar. 


\subsection{Initial Jet Tip Vortices}

Figures 5-6 show the initial stages of the hydrogen jet (NPR=8.5, 10 respectively) penetrating into the chamber with $P_{\infty} \approx 1$ bar. Specifically, these figures present mass fraction contours and the line integral convolution [55] of velocity vectors for a period up to $t \approx 16 t_{0}$. It can be seen that the spherical bow shock in front of the jet boundary propagated with relatively high velocity magnitudes ranging $600-1000 \mathrm{~m} / \mathrm{s}$. The air in front of the jet boundary (see mass fraction contours) accelerated because of the pressure waves emitted from the jet. The speed of the air upstream was higher than the speed of sound in air with the initial ambient temperature (of order $300 \mathrm{~m} / \mathrm{s}$ ). This was due to rapid compression which could increase the temperature of the air ahead to values of $500-600 \mathrm{~K}\left(\mathrm{H}_{2}\right.$ with $\mathrm{NPR}=8.5-10, P_{\infty} \approx 1$ bar $)$. Temperature rise would increase the speed of sound significantly. This rapid acceleration resulted in comparable velocity magnitudes of the jet tip and its adjacent air, as seen in the velocity fields of Figures 5-6 where the $\mathrm{H}_{2}$ jet tip cannot be clearly identified; at the boundary of the jet and ambient air there is a contact discontinuity [56]. The flow in front of the bow shock was almost stationary. This spherically propagating shock could have significant influence on the mixing process by enhancing air entrainment.

Figures 5-6 also show another important feature of the transient under-expansion process, the tip vortices. This type of behaviour normally occurs when a low density gas is issued into a high density environment or a cold gas is injected into hot ambient [56-57]. This feature of enhanced turbulent mixing through developing vortical flows plays an important role in the mixing process of under-expanded jets. It is worth mentioning that the tip vortex is a three dimensional toroidal feature, often called 'vortex ring', and may include various types of vortices [53]. The tip vortex shown in Figures 5-6 formed in the course of rolling up of the jet's turbulent interface close to the edge of the near-nozzle shock structure; see snapshots at $t \approx 16.1 t_{0}$ of the integral convolution. The hydrogen jets showed fairly similar transient behaviour of their tip vortices. However, the initial sign of tip vortex formation appeared earlier for the jet with higher NPR. Specifically, the early tiny tip vortices appeared at $t \approx 9.6 t_{0}$ and $t \approx 11.3 t_{0}$ for NPR=10 and 8.5 , respectively.

Similarly to those figures, the transient formation process of the tip vortices for the hydrogen jet with NPR $=10$ issued into air of $P_{\infty} \approx 10$ bar is presented in Figure 7. It was found that the jets with analogous NPR exhibited fairly similar initial transient evolution for their tip vortices. Also comparison of the initial penetration of jets with similar NPR but different ambient pressures showed almost identical behaviour in Figures 6-7. However, slightly higher maximum velocity was noticed for the jet injected into $P_{\infty} \approx 1$ bar and the tip vortices recirculated slightly faster compared to the jet injected into $P_{\infty} \approx 10$ bar, e.g. compare the $t \approx 12.9 t_{0}$ snapshots in Figures 6-7. On the other hand, greater hydrogen mass fraction was observed within the recirculation area of the jet injected into $P_{\infty} \approx 10$ bar. Therefore, it may be summarised that the relatively faster tip recirculation of the jet with $P_{\infty} \approx 1$ bar was because of the lower mass within its recirculation area compared to that of the jet with $P_{\infty} \approx 10$ bar. An important conclusion from Figures 5-7 is that for a particular gas the transient evolution of the tip vortices is mostly affected by NPR rather than the level of the incoming momentum (i.e. injection pressure). 
Similar type of tip vortex formation was also noticed in [53] for an under-expanded nitrogen jet, albeit with the vortices shifted noticeably further downstream compared to the hydrogen jets of the current study. This could be associated with the higher nozzle exit velocity of hydrogen $(\sim 1300 \mathrm{~m} / \mathrm{s})$ compared to that of nitrogen $(\sim 330 \mathrm{~m} / \mathrm{s})$. In order to clarify this, the initial transient steps of the methane jet (NPR=8.5, $P_{\infty} \approx 1$ bar) were investigated as shown in Figure 8. For this condition the methane jet exited from the nozzle with a velocity of $U_{1} \approx 450 \mathrm{~m} / \mathrm{s}$ which was much closer to that of nitrogen in [53] than hydrogen here. Similarly to [53], Figure 8 reveals that, unlike hydrogen, the methane jet exhibited a relatively smaller initial flow recirculation which consequently formed the jet tip vortices after the Mach disk location. The temporal intervals in Figures 5-8 are based on $t_{0}$ which was $\sim 3$ times larger for the methane jet than that of hydrogen. Therefore, it is clear that the jet tip vortices were formed considerably earlier for hydrogen than for methane. For the hydrogen jet with NPR=8.5 the fully recirculating tip vortices were observed within $7.0 \mu$ s whereas for the respective methane jet these vortices were noticed within $20 \mu \mathrm{s}$. The mass flow rate of the methane jet with NPR $=8.5$ was $2.3-3.0$ times higher than those of the hydrogen jets with NPR $=8.5$ and 10 . Therefore, the difference in the evolution of the tip vortices can only be an effect of the significant differences in nozzle exit velocity between the methane and hydrogen jets. The higher velocity magnitude of hydrogen would also produce a noticeably higher radial velocity compared to that of methane. As presented in Figure 8 for methane, a tiny recirculation was formed at $t \approx 5.5 t_{0}$. However, the local velocity was not high enough to form a dominant vortex and, in turn, this initial recirculation moved downstream and got linked to the jet's penetration. However, at $t \approx 11.1-13.8 t_{0}$, the tip recirculation became relatively strong and formed noticeable tip vortices downstream of the Mach disk.

The aforementioned difference in the tip vortex evolution between methane and hydrogen jets may affect significantly the fuel-air mixing upstream of the Mach disk location. The current authors found previously that, unlike hydrogen, methane did not exhibit any mixing before the Mach disk (at the boundary of the intercepting shock) [14]. Based on this and also some other relevant observations [13,37, 40], it may be concluded that the relatively high level of turbulence at the nozzle exit of hydrogen jets triggered the Görtler vortices [58] and thus increased the instability at the boundary of the curved intercepting shock. However, the velocity fields in Figures 5-8 also show that velocity magnitudes as high as $U \approx 1600 \mathrm{~m} / \mathrm{s}$ can exist at the boundary of the intercepting shock (upstream of the Mach reflection) in hydrogen jets due to the presence of strong tip vortices. Therefore, it can be further concluded that, in addition to the Görtler-vortices-induced instabilities, the transient tip vortices may also contribute significantly to the flow instabilities at the boundary of the intercepting shock and promote hydrogen-air mixing before the Mach disk location.

It is noted here that comparable behaviour was found between the evolution of the transient near-nozzle shock structure and transient vortex ring of under-expanded jets between the current second-order accurate STAR-CCM $+{ }^{\circledR}$ framework and the aforementioned newly developed fourth-order accurate OpenFOAM ${ }^{\circledR}$ solver [51]. Figures A.1-A.2 in the Appendix show a direct comparison between the two computational frameworks for an under-expanded nitrogen jet with NPR=8.5 issued into a nitrogen ambient with $P_{\infty}=1$ bar. In addition to the order of the temporal discretization, the turbulence model (one-equation LES model for the 
OpenFOAM $^{\circledR}$ solver) and the flux-splitting method [51] were also different features between the two simulations. Although very close jet behaviour was captured by both codes, the OpenFOAM ${ }^{\circledR}$ solver with higher order accuracy was able to provide a sharper representation of the shock structure and vortex ring rolling-up process.
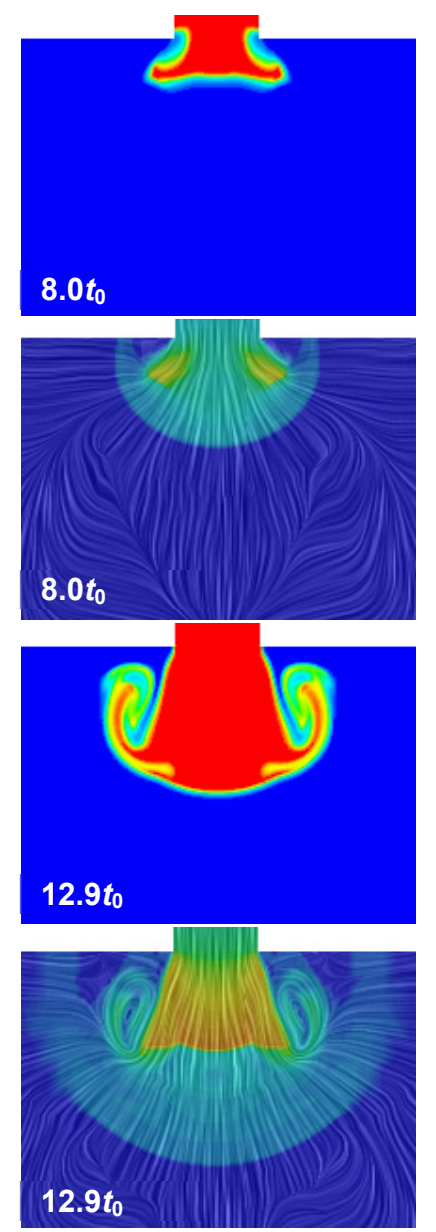
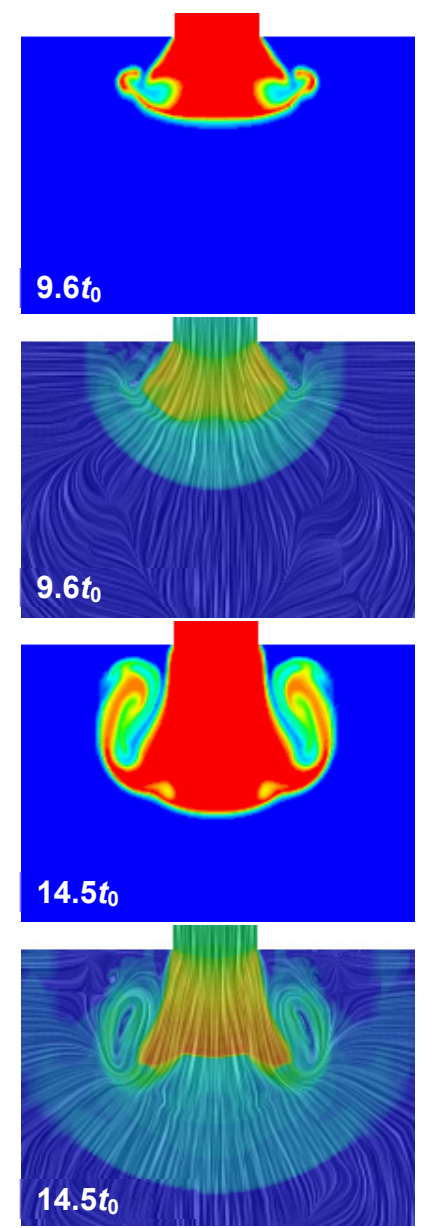
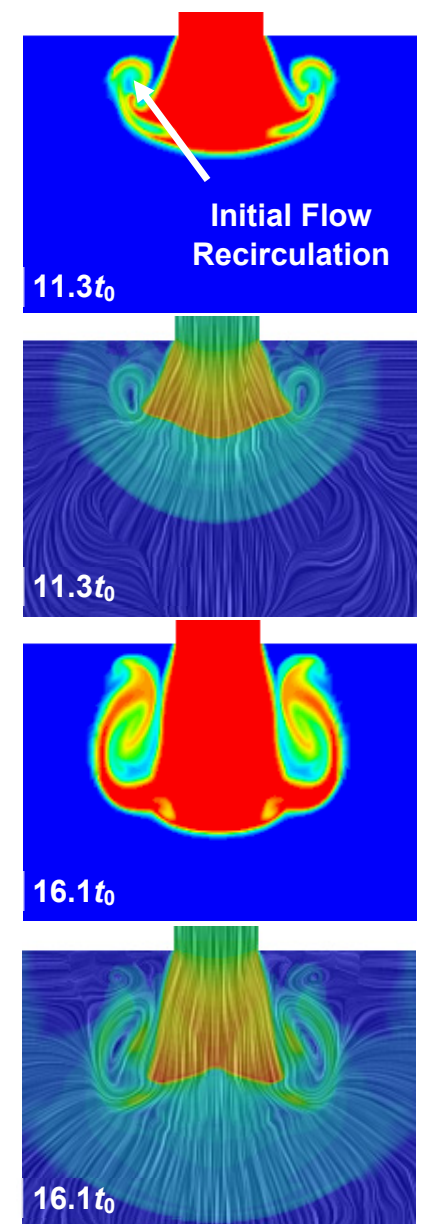

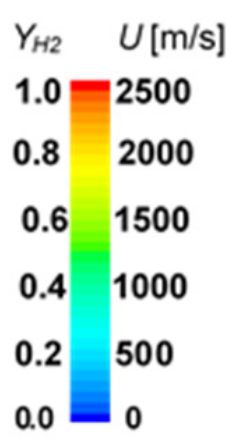

$\begin{array}{ll}Y_{H 2} & U[\mathrm{~m} / \mathrm{s}] \\ 1.0 & 2500 \\ 0.8 & 2000 \\ 0.6 & 1500 \\ 0.4 & 1000 \\ 0.2 & 500 \\ 0.0 & 0\end{array}$

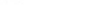

Figure 5. Transient jet development of hydrogen $\left(\mathrm{NPR}=8.5\right.$ and $P_{\infty} \approx 1$ bar) tip vortices $\left(t_{0} \approx 6.2 \cdot 10^{-7}\right)$. First and third rows: $\mathrm{H}_{2}$ mass fraction $\left(Y_{\mathrm{H} 2}\right)$; Second and fourth rows: Line integral convolution of the velocity vectors. 

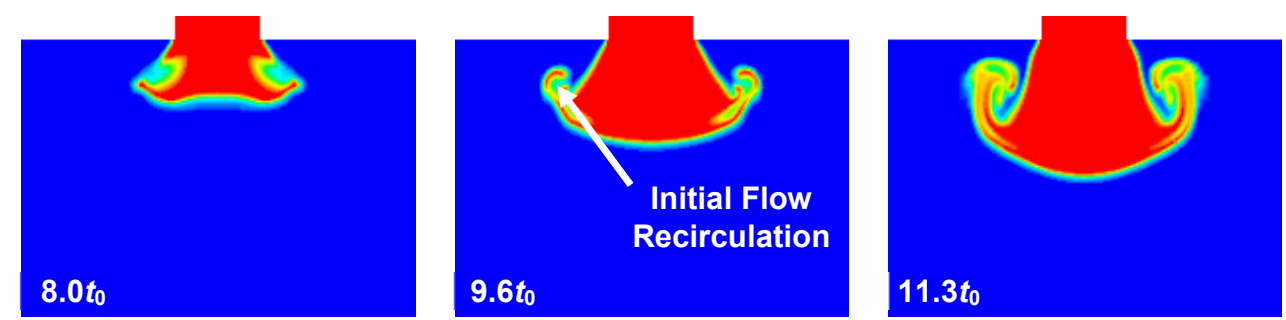

$Y_{H 2} \quad U[\mathrm{~m} / \mathrm{s}]$

$1.0-2500$

$0.8 \quad 2000$

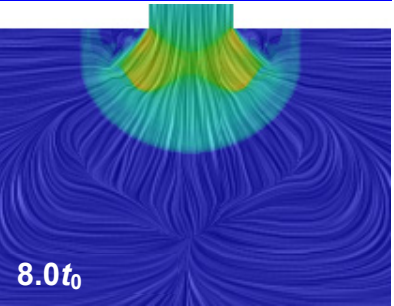

$9.6 t_{0}$
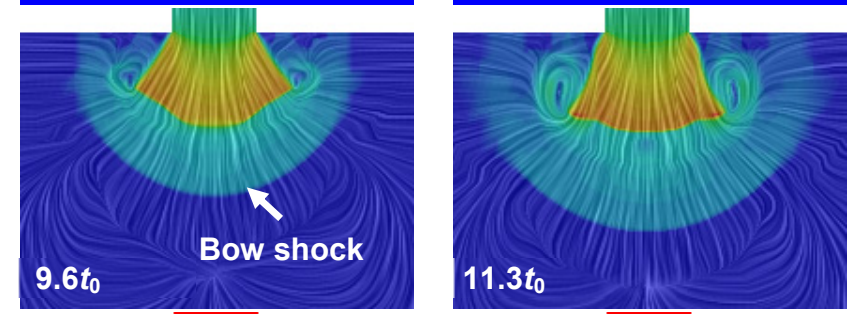

0.61500

0.41000

$0.2 \quad 500$

$0.0=0$
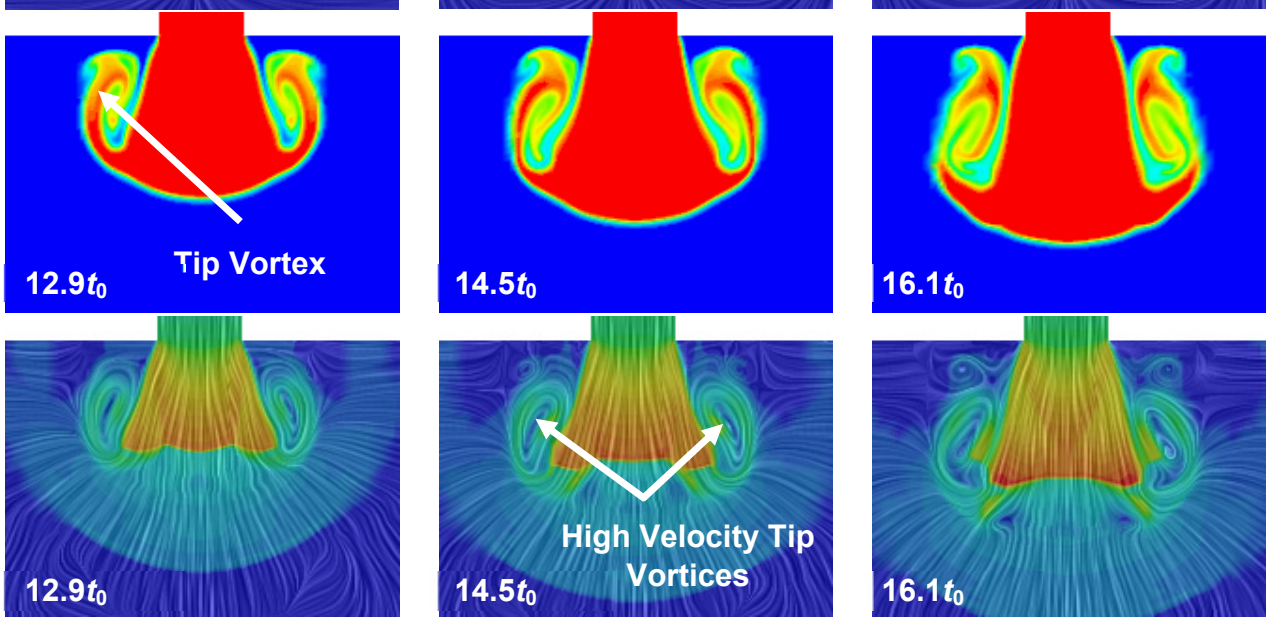

Figure 6. Transient jet development of hydrogen (NPR=10 and $P_{\infty} \approx 1$ bar) tip vortices $\left(t_{0} \approx 6.2 \cdot 10^{-7}\right)$. First and third rows: $\mathrm{H}_{2}$ mass fraction $\left(Y_{\mathrm{H}_{2}}\right)$; Second and fourth rows: Line integral convolution of the velocity vectors. 

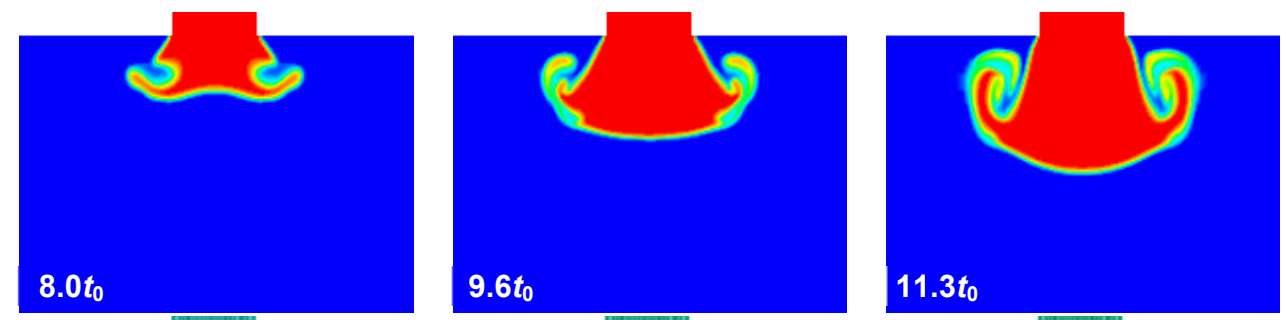

$$
\begin{array}{ll}
Y_{H 2} & U[\mathrm{~m} / \mathrm{s}] \\
1.0 & 2500 \\
0.8 & 2000 \\
0.6 & 1500 \\
0.4 & 1000 \\
0.2 & 500 \\
0.0 & 0
\end{array}
$$
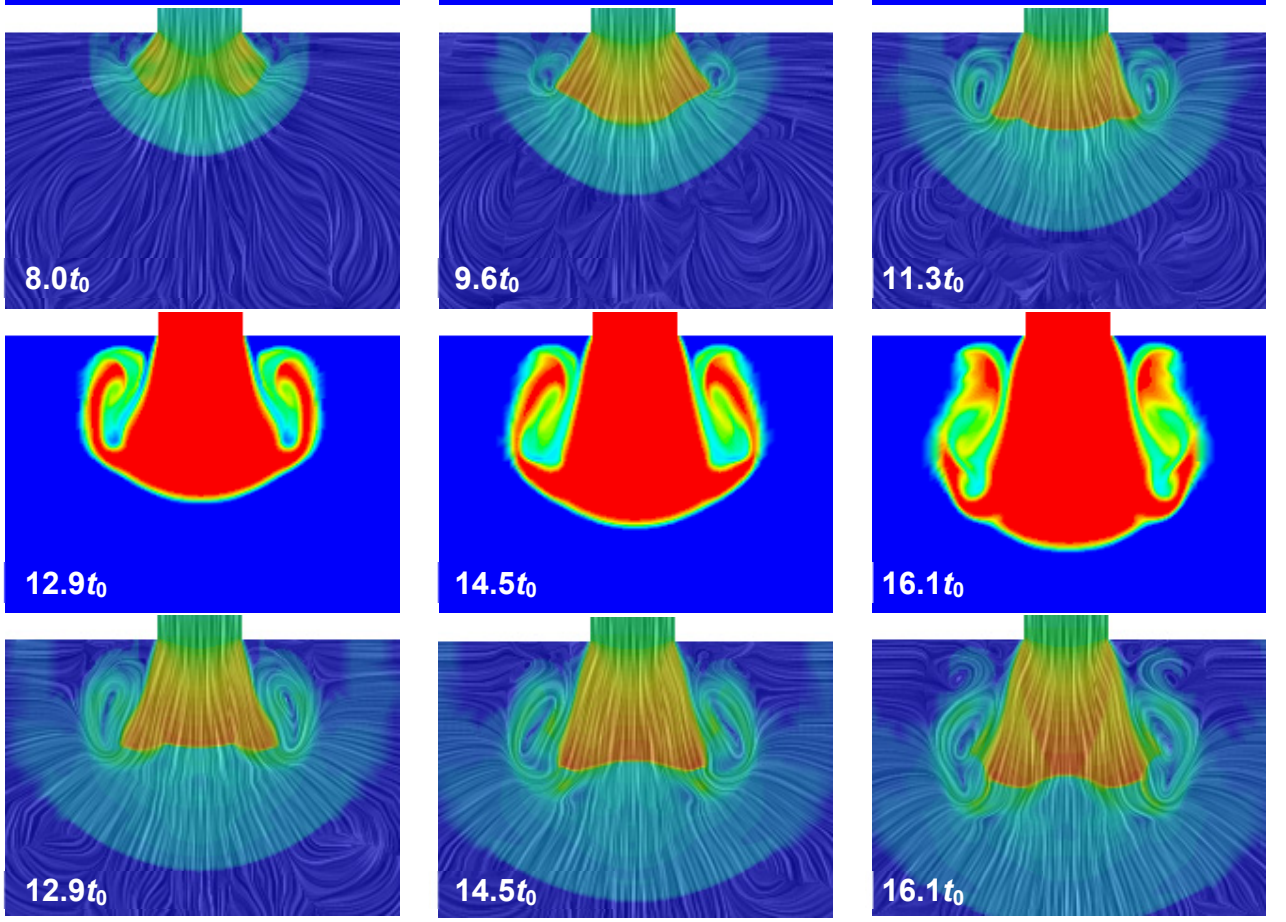

Figure 7. Transient jet development of hydrogen $\left(\mathrm{NPR}=10\right.$ and $P_{\infty} \approx 10$ bar) tip vortices $\left(t_{0} \approx 6.2 \cdot 10^{-7}\right)$. First and third rows: $\mathrm{H}_{2}$ mass fraction $\left(Y_{\mathrm{H}}\right)$; Second and fourth rows: Line integral convolution of the velocity vectors. 

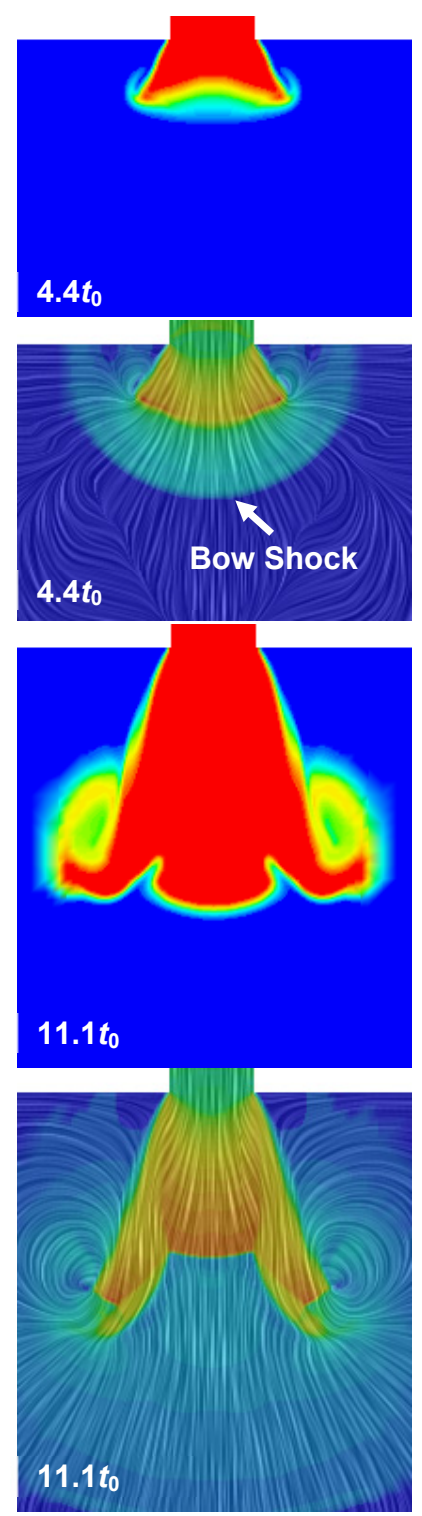
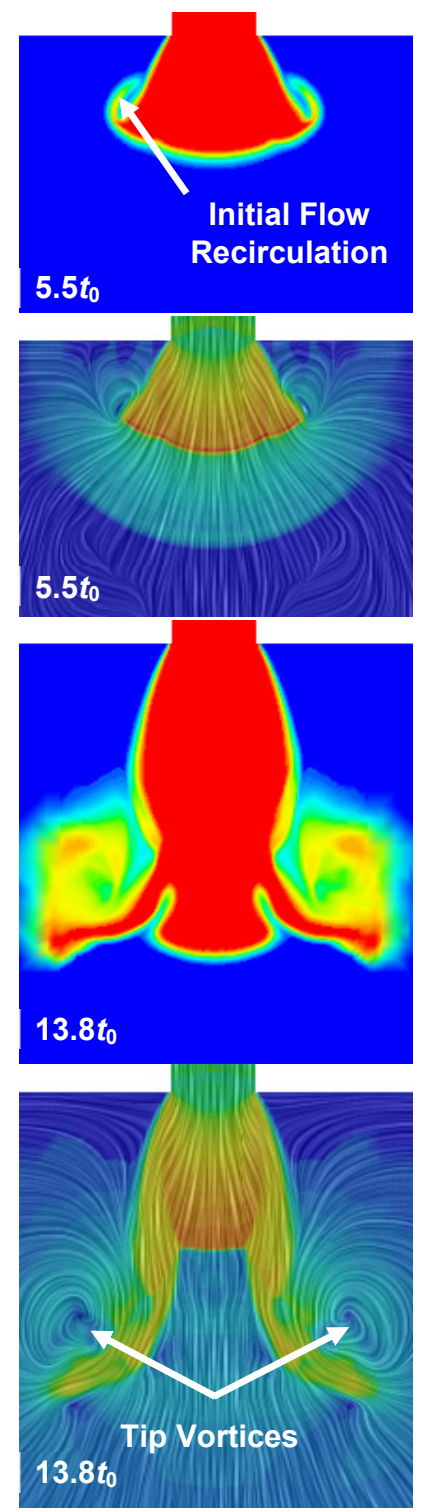
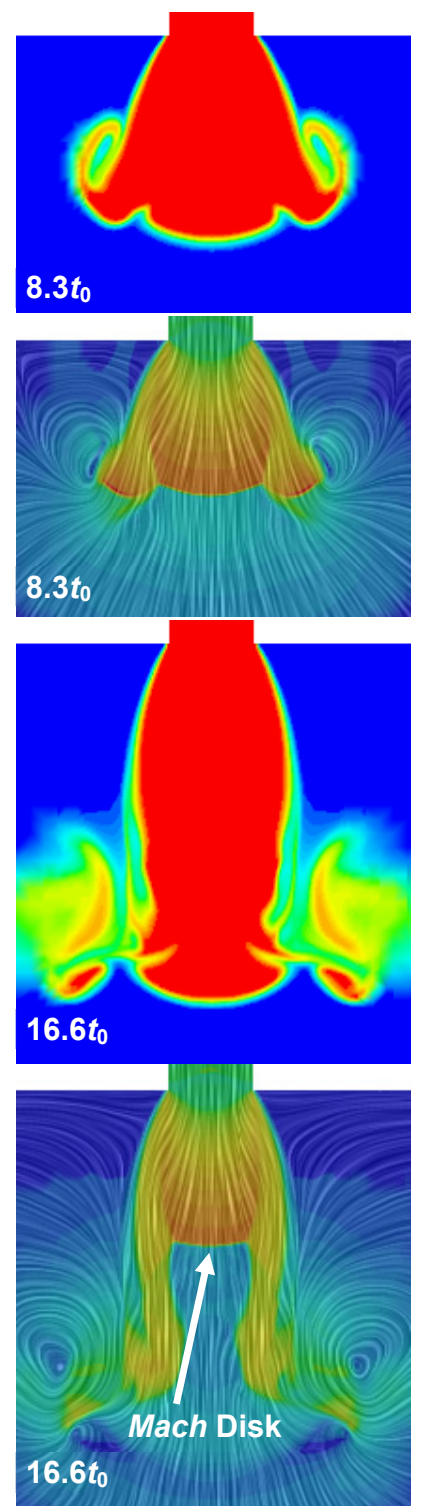

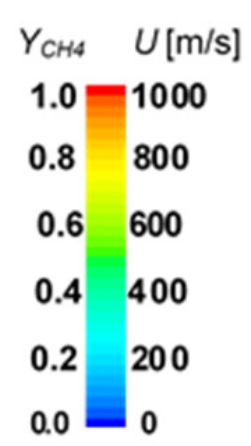

$$
0.0-0
$$

Figure 8. Transient jet development of methane (NPR=8.5 and $P_{\infty} \approx 1$ bar) tip vortices $\left(t_{0} \approx 1.8 \cdot 10^{-6}\right)$. First and third rows: $\mathrm{CH}_{4}$ mass fraction $\left(Y_{\mathrm{CH}}\right)$; Second and fourth rows: Line integral convolution of the velocity vectors.

\subsection{Quasi-Steady Shock Structure}

\subsubsection{Near-Nozzle Shock Characteristics}

In order to obtain better understanding of the near-nozzle shock structure in under-expanded jets it is important to study this region under semi-steady conditions. Figure 9 shows a direct comparison between the near-nozzle shock structure of hydrogen and methane jets (NPR=8.5 and $P_{\infty} \approx 1$ bar) at $t \approx 161 t_{0}$. The nearnozzle structure reached a semi-steady condition far before this time. The transverse and axial velocity profiles of the methane and hydrogen jets on the vertical plane of Figure 9 are illustrated in Figure 10. The red dashed lines in Figure 9 show the border of the inner and outer shear layers. The dashed ovals in the same figure show the origin where the outer shear layer starts forming. It is clear that for hydrogen the origin of the outer shear layer was close to the boundary of the intercepting shock at about half Mach disk height downstream of the nozzle exit. For methane, this origin was located after the Mach disk. Direct comparison 
between Figure 10 and Figures 5 and $\mathbf{8}$ revealed that the location of the centres of the dashed ovals were very similar to the centroids of the initial strong jet tip vortices.

Based on these observations a mechanism is proposed for the start of the mixing process in under-expanded jets. Specifically, after the formation of a complete recirculation (see the last velocity frames of Figure $\mathbf{5}$ and Figure 8) the tip vortices became almost stationary while the main stream of the jet continued penetrating. This would cause entrainment of ambient air into the main stream of the emerging hydrogen jet and also 'separation' of part of the hydrogen jet from its main axial stream and mixing with the surrounding air. This initial mixing forms the mixing layer or the outer shear layer in under-expanded jets. However, the strength of the almost stationary vortices decreased gradually and after some time these vortices vanished entirely. The proposed mixing mechanism can also be linked to an explanation of the mixing process past this time. Further investigation showed that at $t \approx 161 t_{0}$, within the location of the former tip vortices in the hydrogen jet (i.e. those that vanished completely), the jet velocity was as high as $\mathrm{U} \approx 150 \mathrm{~m} / \mathrm{s}$ and remained within this order for the $0.3 \mathrm{~ms}$ injection duration studied. Therefore, the proposed mixing mechanism can be completed by considering the effect of this high velocity magnitude on the development of the Görtler-type instabilities. The Görtler-type instabilities contributed significantly to the formation of the outer shear layer in hydrogen jets. In contrast, for methane, due to the final localisation of the tip vortices (after the Mach disk) the formation of the shear layer was affected by the Mach reflection.

From Figure 9 it can be observed that for both methane and hydrogen, just after the Mach disk, the subsonic core of the jet (i.e. the volume surrounded by the slip lines) initially expanded and then contracted rapidly. Since there was no pressure disparity across the slip line, this expansion and contraction behaviour did not occur due to pressure-related mechanisms. This behaviour has been explained in [10] through a set of schlieren and PIV images and was attributed to the direction of the transverse component of the velocity field. Similarly to [10,59], the transverse velocity graphs of Figure 10 show that just after the Mach disk location $(\mathrm{Z} / D \approx 1.9$ and 1.85 for methane and hydrogen, respectively), at $2.0 D$ downstream of the nozzle exit, the flow has a transverse velocity component towards the jet boundary (positive value of $u / U_{1}$ ). This was attributed to the relative concavity of the Mach disk (see Figure 9) due to the initial expansion fans at the nozzle lip [10]. However, after a radial distance of $\sim 0.55 D$, the flow was turned inwards by passing through an oblique shock which was formed by the reflection of the shock at the triple point. This resulted in a transverse velocity component towards the jet centreline. This inward flow became dominant within the volume of the inner shear layer at a distance $2.0 D-2.2 D$ which then caused the contraction of the jet core.

The transverse profiles of Figure 10 show that, for both methane and hydrogen, the jet flow had a transverse velocity component towards the boundary for distances upstream of the Mach disk. The normalized values of these transverse velocities $\left(u / U_{1}\right)$ were higher for methane than for hydrogen. Figure $\mathbf{1 0}$ also shows that for distances away from the nozzle centreline the magnitude of the transverse velocity component increased. Therefore, it may be concluded that the concavity of the Mach disk is due to gradient of the transverse velocity component (see the lines of $Z / D=1.8$ for the transverse profiles of Figure 10), not directly by the initial expansion fans at the nozzle lip as suggested in [10]. In fact, by moving away from the nozzle 
centreline, the direction of the velocity vector produced by the transverse and axial velocity components would be more inclined with respect to the nozzle centreline. This can also explain the existence of a more concave Mach disk in the methane jet compared to the hydrogen jet as presented in Figure 9. Due to the higher gradient of the transverse velocity component just before the Mach disk in the methane jet when compared to that of hydrogen jet (see $Z / D=1.8$ in Figure 10), the aforementioned vector was more inclined away from the nozzle centreline of the methane jet (particularly close to the triple points), which consequently resulted in relatively more concave Mach disk. The axial velocity profile of Figure 10 shows how the flow accelerated and decelerated (axial component of velocity increased and decreased) upstream and downstream of the Mach reflection. Also, the aforementioned axial profiles can be used to approximate the thickness of inner and outer shear layers. Each axial profile of Figure 10 has a section with almost constant $w / U_{1}$ (almost vertical lines for $w / U_{1}>1$ and $\mathrm{Z} / D>1.8$ ). It was found that the length of these almost vertical sections represented practically $80-90 \%$ of thickness of the inner shear layer (depending on jet condition and downstream location). On the other hand the thickness of the outer shear layer can be estimated as the distance between the highest $\mathrm{X} / D$ of the aforementioned vertical section of the axial velocity profiles (Figure 10) and where $w / U_{1}$ turns into nil.

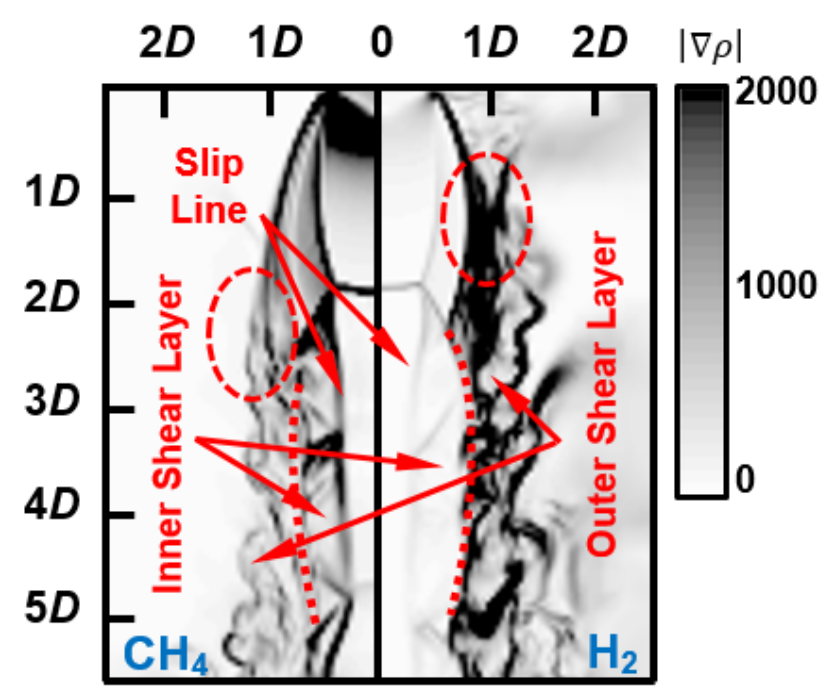

Figure 9. Semi-steady near-nozzle shock structure at $t \approx 161 t_{0}$ for Methane and Hydrogen jets with NPR=8.5 and $P_{\infty} \approx 1$ bar. 

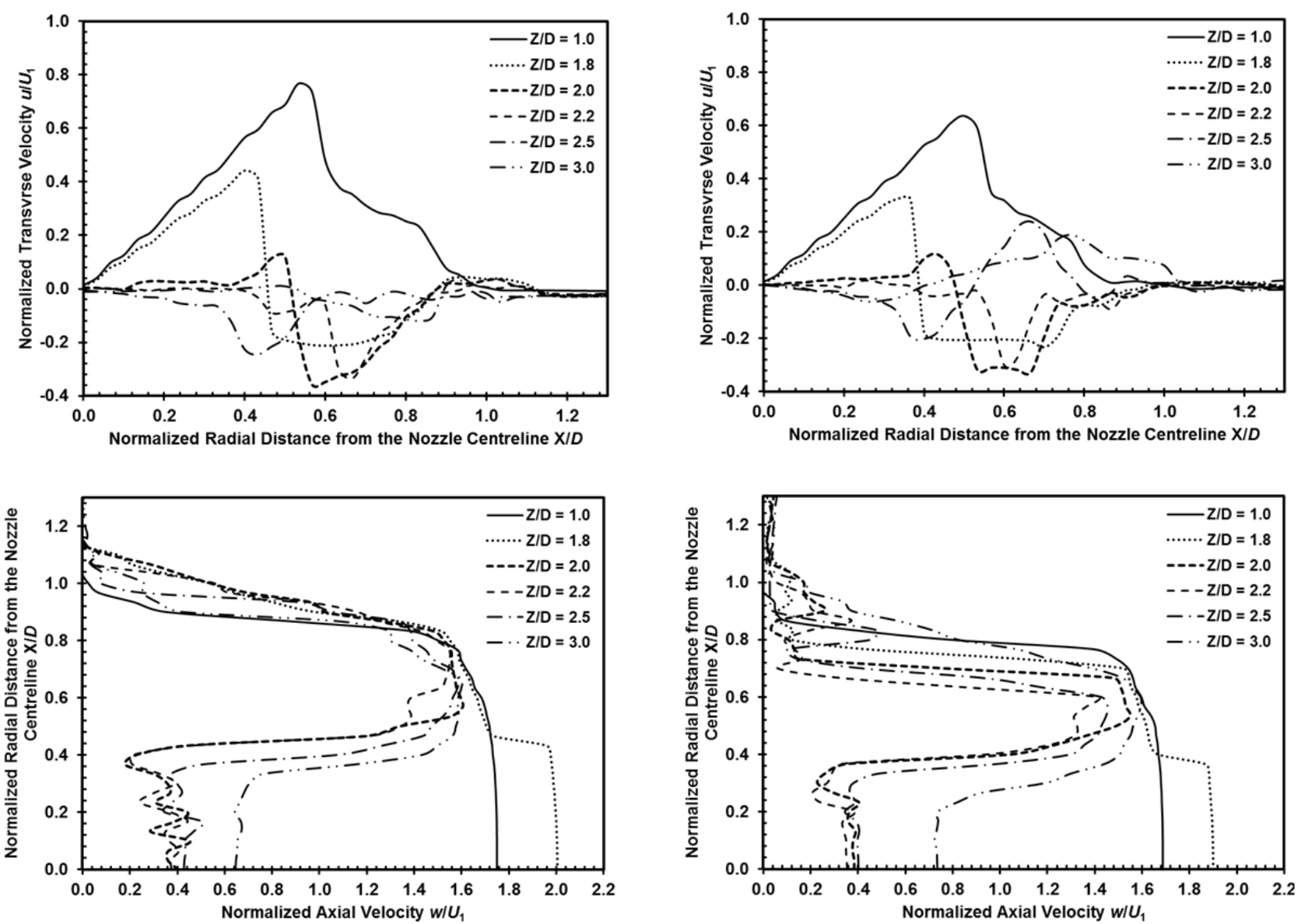

Figure 10. Normalized transverse (top) and axial (bottom) velocity profiles upstream and downstream of the Mach disk for Methane (left) and Hydrogen (right) jets with NPR=8.5 and $P_{\infty} \approx 1$ bar.

\subsubsection{Mach Reflection Recirculation Zone}

To date all numerical studies based on the Navier-Stokes equations have detected recirculation zones immediately downstream of the Mach disk [10, 60-61]. However, numerical modelling studies using Monte Carlo methods [62] and also experimental observations [10, 63] have shown no evidence for such flow behaviour. For instance, in PIV measurements conducted by Edgington-Mitchell et al. [10] on an underexpanded air jet with $\mathrm{NPR}=4.2(D=15 \mathrm{~mm})$ no recirculation zone behind the Mach disk was recorded. Breakdown of the continuum assumption of Navier-Stokes equations in the vicinity of strong shocks like the Mach disk has been suggested as a possible reason for previously observed flow recirculation just downstream of the Mach disk [10]. It has been shown [63] that Mach reflection could not produce the recirculation zone and only a 'cap-shock' pattern with adequate curvature might be able to produce a trapped vortex or recirculation behind a small normal shock. As shown in Figure 11 the current LES study, in excellent agreement with previous experimental observations [10,63], did not capture any kind of flow recirculation downstream of the Mach disk in under-expanded jets. However, in all previous numerical studies [60-61] flow recirculation was observed within the area surrounded by the red oval in Figure 11. It is worth mentioning that all hydrogen and methane under-expanded jets of the current study exhibited similar behaviour to what presented in Figure 11. As further confirmation to what was concluded in [10], and based 
on the current high fidelity study, the flow recirculation downstream of the Mach disk is believed to be almost certainly an artefact of the types of numerical approach previously used to study under-expanded jets by other authors. It is also noted that in agreement with the current STAR-CCM $+{ }^{\circledR}$ framework the aforementioned high order accurate OpenFOAM ${ }^{\circledR}$ solver also did not produce any kind of flow recirculation zone downstream of the Mach reflection [51].

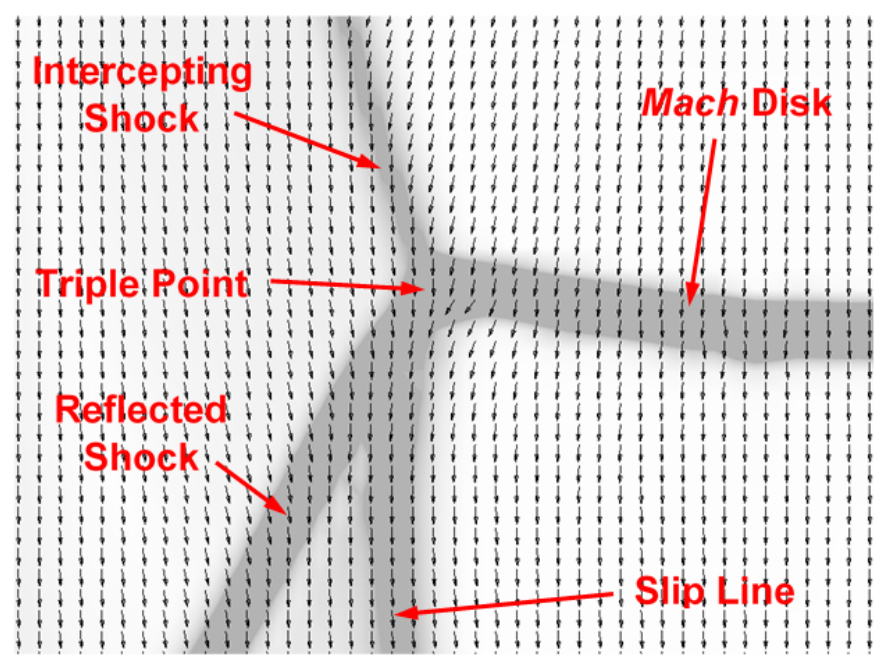

Figure 11. Velocity vectors and shock structure at the vicinity of the triple point in under-expanded methane jet with NPR=8.5 and $P_{\infty} \approx 1.0$ bar.

\subsection{Flow Characteristics}

Figure 12 compares contours of density gradients in hydrogen jets with NPR=10 issued into ambient with pressures $P_{\infty} \approx 1$ bar, 5 bar and 10 bar.
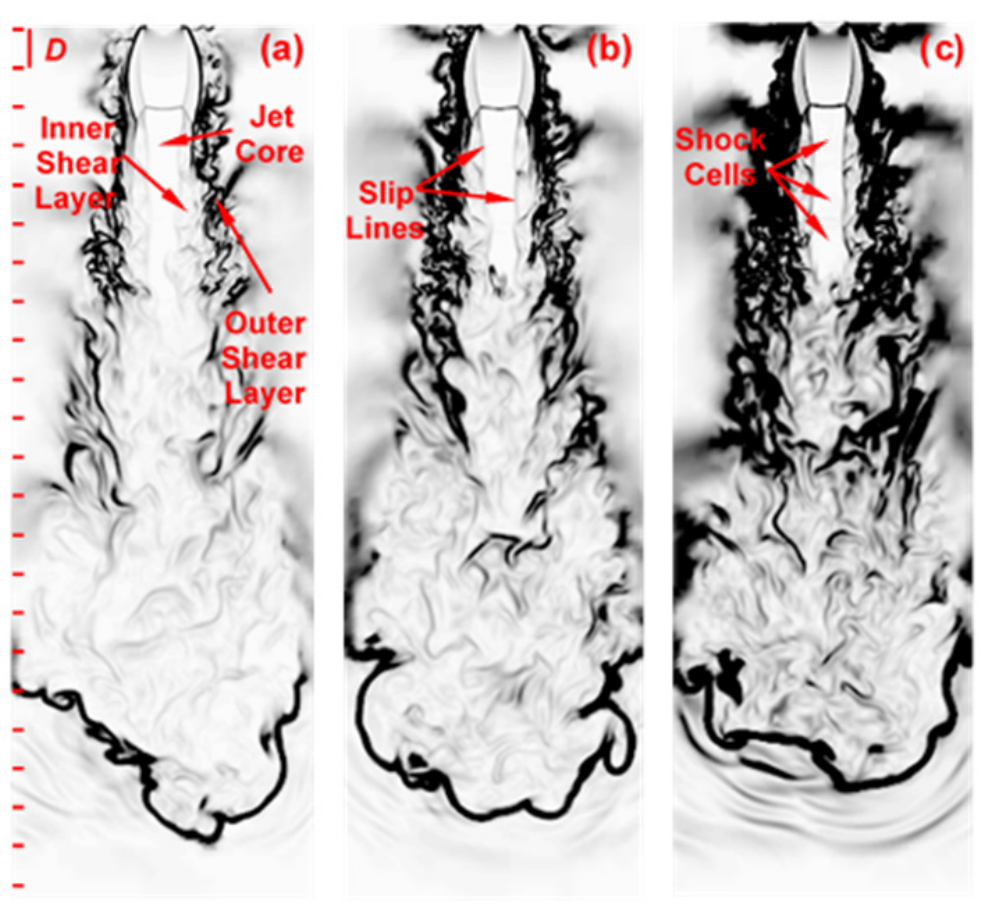

Figure 12. Density gradient $|\nabla \rho|$ field of the under-expanded $H_{2}$ jets with $N P R=10$ and (a): $P_{\infty} \approx 1$ bar, $(b): P_{\infty} \approx 5$ bar and $(c): P_{\infty} \approx 10$ bar $\left(t \approx 161 t_{0}\right)$. 
The greyscale legend of Figure 12 is similar to that of Figure 9 but with different upper scales for visualisation enhancement. Specifically, the upper limit for the jet with $P_{\infty} \approx 1$ bar is $|\nabla \rho|=2000$ and for the other jets is $|\nabla \rho|=6000$. This is due to the higher level of density gradient for the cases with higher ambient pressures which would be masked if the same scale was used as (existence of large zones with extremely dark scales was avoided). The effect of the legend limits on the visualisation quality of density gradient contours in under-expanded jets has been discussed comprehensively earlier and not repeated here for brevity $[14,35]$.

Figure 12 shows that with constant NPR and regardless of ambient pressure, hydrogen exhibited almost identical shock characteristics, particularly in terms of Mach disk dimensions. A value of $C_{H} \approx 0.65$ for the constant of Equation (1) was calculated for the jets with NPR $=10$ under elevated ambient pressures $\left(P_{\infty} \approx 5\right.$ bar and 10 bar), similarly to the $\mathrm{H}_{2}$ jet with $\mathrm{NPR}=10$ and $P_{\infty} \approx 1$ bar. Significantly higher level of momentum exchange (density gradient) was observed for the jets injected into elevated ambient pressure. This can be seen from the extremely dark regions in the contours of density gradient presented in Figure 12. This was attributed to the fact that the under-expanded jets injected into the elevated ambient pressures had fairly higher level of momentum (density) at the nozzle exit (to maintain a constant NPR). Therefore, although the diffusivity of hydrogen decreased noticeably under elevated pressures, the higher level of incoming momentum enhanced the hydrogen-air mixing particularly at the jet boundary after the location of the Mach disk. The jet with $P_{\infty} \approx 5$ bar showed slightly wider half cone angle $\left(\sim 3^{\circ}\right.$ wider) and $\sim 4 \%$ lower penetration (at $t \approx 161 t_{0}$ ) compared to its counterpart injected into $P_{\infty} \approx 1$ bar with the same NPR of 10 . This can be explained by the relatively higher level of radial momentum and hence radial mixing for the jet issued into elevated pressure conditions. As discussed earlier (see Figures 6-7), relatively similar jet tip vortices in terms of velocity characteristics can exist for under-expanded jets under various ambient pressures but at fixed NPR. However, the tip vortices of the jet issued into elevated pressure conveyed more mass because of the higher injection pressure. Therefore, this jet transports relatively greater level of radial momentum which can result in fairly larger radial penetration and jet cone angle. For the same NPR, the hydrogen jet injected into the ambient with $P_{\infty} \approx 10$ bar penetrated $\sim 3 \%$ less than that of the jet issued into $P_{\infty} \approx 5$ bar (at $t \approx 161 t_{0}$ ).

Instantaneous snapshots of spatial variations of various flow quantities at $t \approx 161 t_{0}$, including $\mathrm{H}_{2}$ mole fraction, temperature, velocity, Mach number and vorticity for the hydrogen jets with NPR=10 are presented in Figure 13 for all ambient pressures of 1, 5 and 10 bar. From this figure it is clear that due to the rapid expansion of the jet, the velocity and the Mach number reached maximum values of $2540 \mathrm{~m} / \mathrm{s}$ and 3.98 , respectively, in the vicinity of the Mach reflection. The values of centreline Mach number and density at $t \approx 161 t_{0}$ for the hydrogen jet with NPR=10 are plotted in Figures 14-15 and are compared to those with $\mathrm{NPR}=30$ and 70 with $P_{\infty} \approx 1$ bar studied previously in [14]. It is clear from Figure 14 that for all values of NPR, the Mach number at the nozzle exit was $\sim 1.1$. The maximum value of Ma occurred in the vicinity of the Mach disk and was 3.94, 5.36 and 6.60 for NPR values of 10, 30 and 70, respectively. In Figure 14 it can be seen quantitatively that the NPR is the only influential factor on the Mach number distribution and, in general, on the sonic characteristics of under-expanded jets. The nozzle exit velocity for all values of NPR 
was about $1310 \mathrm{~m} / \mathrm{s}$. Values of the nozzle exit density in Figure 15 show that the jets issued into elevated air pressures had significantly greater density at the nozzle exit compared to that issued into $P_{\infty} \approx 1$ bar ambient. The values of density at the nozzle exit for the $\mathrm{H}_{2}$ jets injected into $P_{\infty} \approx 1$ bar ambient led to mass flow rates of $1.07,3.21$ and $7.50 \mathrm{~g} / \mathrm{s}$ for NPR values of 10,30 and 70, respectively. The calculated mass flow rate for $\mathrm{NPR}=10$ is in good agreement with the value of $\sim 1.0 \mathrm{~g} / \mathrm{s}$ reported by Ruggles and Ekoto [40]; their experimental data were obtained at the same conditions of the current LES study. Figures 12-15 show that the near-nozzle shock structure and sonic characteristics, i.e. Mach disk dimensions, reflected shock angle, velocity and Mach number at the Mach reflection and shock cell spacing for the under-expanded jets with the same NPR are almost identical.

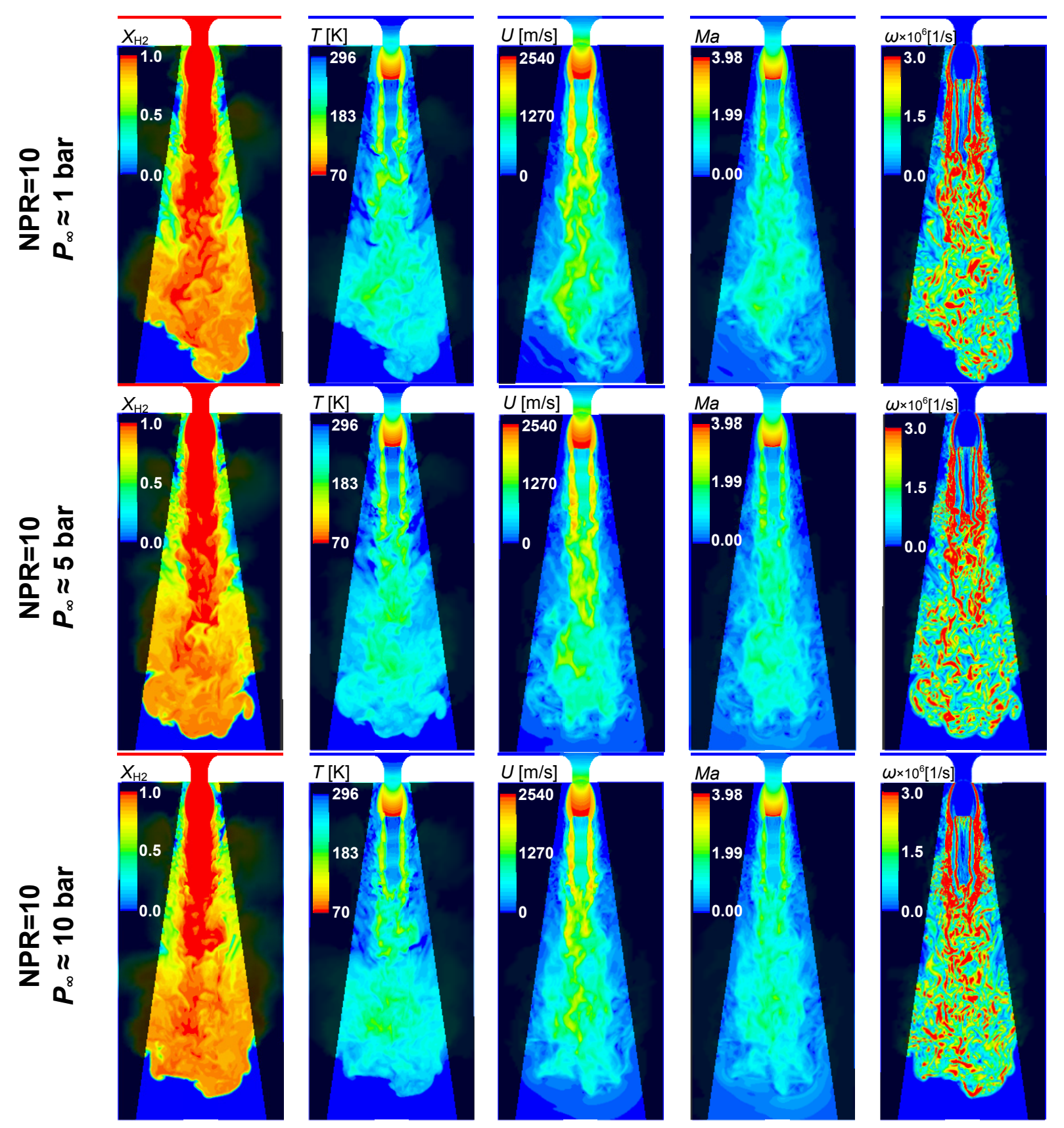

Figure 13. Instantaneous snapshots of various flow quantities in the $\mathrm{H}_{2}$ jets with $\mathrm{NPR}=10$ under various ambient pressures at $t \approx 161 t_{0}$. 
It can be seen in the temperature snapshots of Figure 13 that the predicted values just upstream and downstream of the Mach disk were $\sim 70 \mathrm{~K}$ and $\sim 296 \mathrm{~K}$, respectively (with the latter very close to but lower than the ambient temperature). It is believed that due to the negative Joule-Thomson coefficient of hydrogen, the temperature profile very close to the nozzle exit may not be predicted accurately by the ideal gas equation of state (EoS) employed here. As it has been presented by some researchers [38, 41], for hydrogen jets with extremely high injection pressures $\left(P_{0}>>100\right.$ bar) using a real gas EoS like Redlich-Kwong, resulted in capturing a higher temperature than that of ambient just after the Mach disk. The specific effect of a real gas EoS for the current flow is under on-going study by the current authors and it will be discussed in a future publication. Nevertheless, it has been shown by [38] that for $P_{0}<100$ bar (i.e. relevant to the current study) an ideal gas EoS would produce acceptable temperatures in under-expanded hydrogen jets.

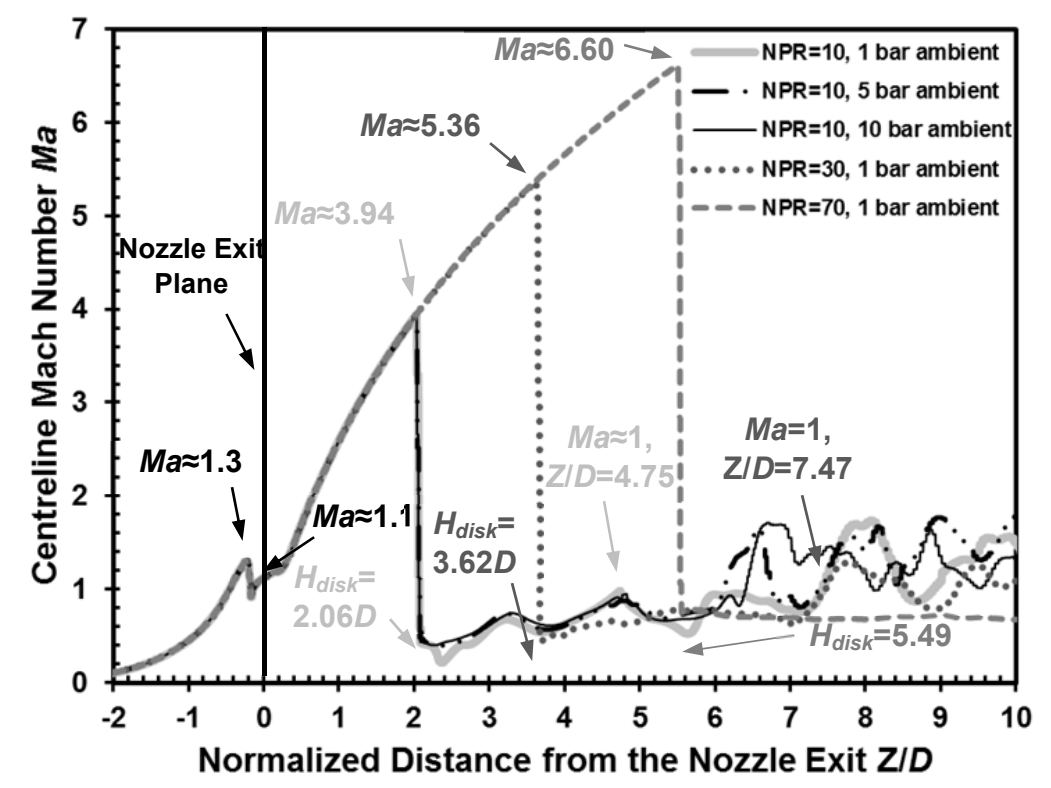

Figure 14. Variation of Mach number in under-expanded $H_{2}$ jets along the nozzle centreline at $t \approx 161 t_{0}$.

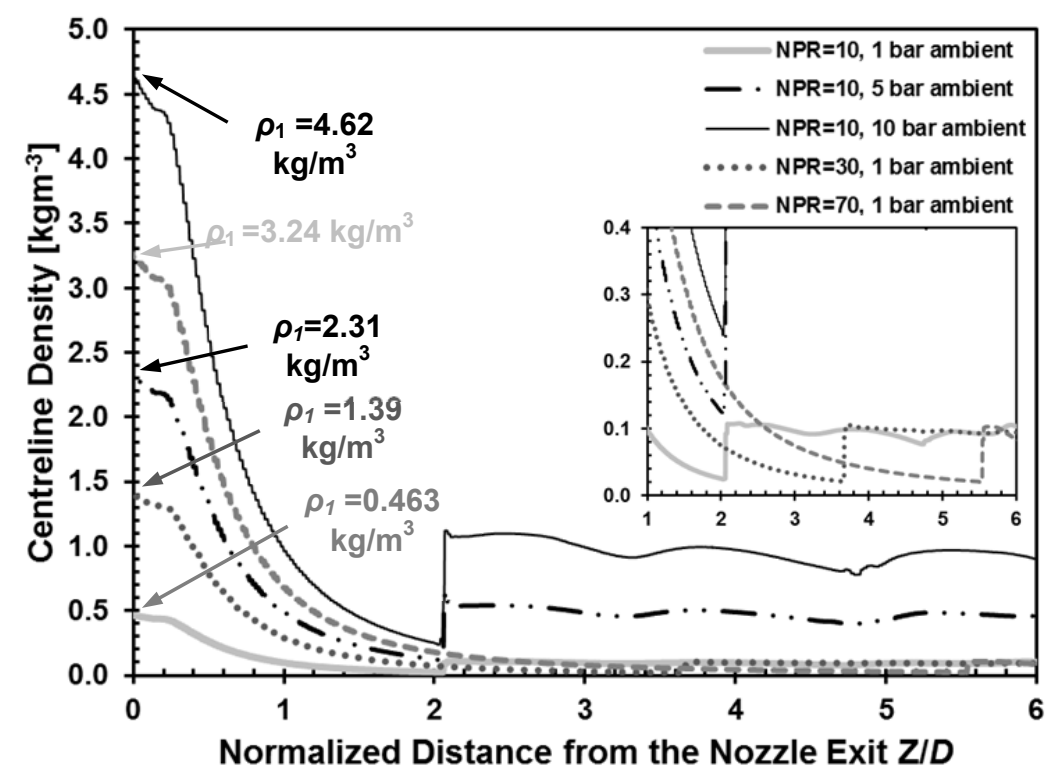

Figure 15. Variation of Density in under-expanded $\mathrm{H}_{2}$ jets along the nozzle centreline at $t \approx 161 t_{0}$. 


\subsection{Shock Cell Spacing}

As discussed in the introduction, shock cell spacing is an important parameter in under-expanded jets and may be used for various purposes. According to Figure 14, the length of the second shock cell was $\sim 4.04$ $\mathrm{mm}$ and $\sim 5.78 \mathrm{~mm}$ for hydrogen jets with $\mathrm{NPR}=10$ and 30, respectively. Also, Figure 13 showed that the maximum Mach number at the Mach disk location was 3.94 and 5.36 for jets with NPR=10 and 30, respectively. If $M a_{j}$ in Equation (3) is assumed to be the Mach number just upstream of the Mach reflection, by rearranging Equation (3), the constant $K$ can be defined as:

$$
K=\frac{L_{\mathrm{s}}}{D \sqrt{M a_{d i s k}^{2}-1}}
$$

If the correlation presented in Equation (9) is solved using data of the hydrogen jets with $\mathrm{NPR}=10$, then a value of $K \approx 0.71$ is achieved. Interestingly, this value can also predict the shock cell length of the hydrogen jet with $\mathrm{NPR}=8.5,30$ and also methane jet with $\mathrm{NPR}=8.5$ with a good accuracy. Therefore, the current work suggests a correlation for the shock cell spacing of under-expanded jets with $8.5 \leq \mathrm{NPR} \leq 30$ as follows:

$$
L_{\mathrm{s}} \approx 0.71 D \sqrt{M a_{\text {disk }}^{2}-1}
$$

As discussed earlier, correlations proposed in the literature based on Equation (3) may not be extended as a general formulation for all types of under-expanded jets [23-25]. Therefore, Equation (10) should be considered only for highly under-expanded jets under comparable NPR and nozzle scale to those used in the present study.

\subsection{Mixing Characteristics}

\subsubsection{Mixture Quality}

Under constant NPR, higher ambient pressure results in slightly wider jet (see Figure 12). Moreover, the mole fraction snapshots of Figure 13 showed that the portion of lean mixture (within the grid refinement area) was reduced gradually by increasing the ambient pressure (and, hence, injection pressure for the same NPR). However, an under-expanded jet has a highly turbulent three-dimensional structure, therefore, the hydrogen-air mixture formation cannot be simply studied by analysis of $2 \mathrm{D}$ contours similar to Figure 13. In this context, the global mixing characteristics of the under-expanded hydrogen jets were examined quantitatively by calculating the probability of a mass weighted function based on the hydrogen mass fraction $\left(Y_{\mathrm{H} 2}\right)$. The probability of $f\left(Y_{\mathrm{H} 2}\right)=\rho Y_{\mathrm{H} 2} \delta_{V}$ was calculated over the computational domain in the refined volume with grid resolution of $D / 50$ and presented in Figure 16. It was found that the hydrogen jet with $\mathrm{NPR}=8.5$ had the lowest probability. It was also found that at constant NPR=10, for hydrogen mass fraction in the range $Y_{\mathrm{H} 2}=0.73-0.93$ (i.e. very rich mixture), higher ambient pressure had higher probability values. Moreover, the integral of $f\left(Y_{\mathrm{H} 2}\right)$ over the full range of mass fraction (0.0-1.0) was higher for the jets with higher NPR and/or higher ambient pressure. All these observations confirm the existence of locally richer mixture for jets with higher NPR or higher ambient pressure at constant NPR. 


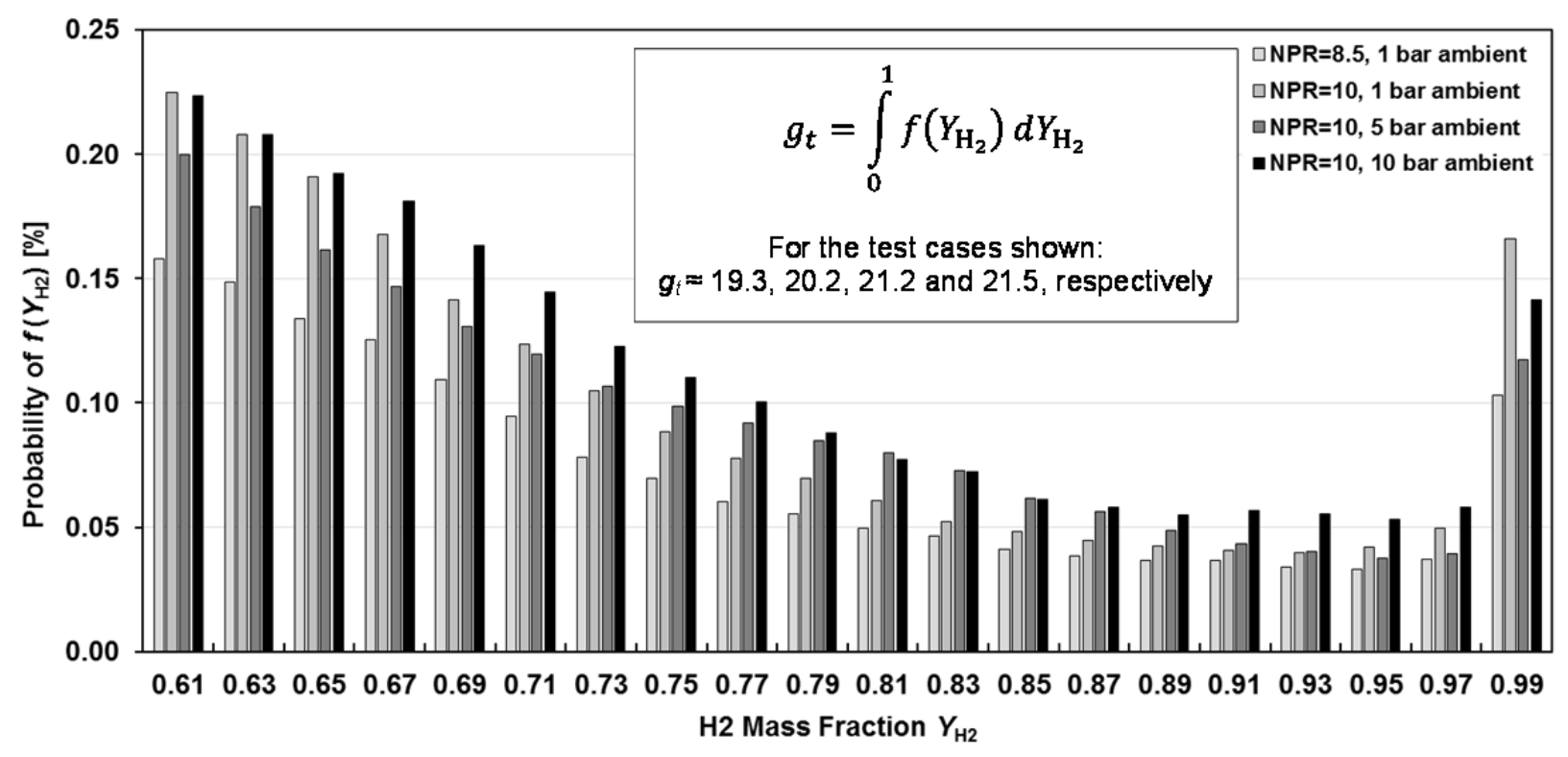

Figure 16. Probability distribution of $\left(\rho Y_{\mathrm{H} 2} \delta_{V}\right)$ within the refinement area with $D / 50$ cell resolution.

Figure 17 compares instantaneous snapshots of hydrogen mole fraction at $t \approx 322 t_{0}$ for jets with $\mathrm{NPR}=10$ under $\sim 1$ bar and $\sim 5$ bar ambient pressure. Figure 17 also shows mole fraction profiles at $Z=15 D$ and $17 D$ downstream of the nozzle exit. From these plots it is now even clearer than in Figure 13 that a generally richer mixture was formed under elevated ambient pressure. Both jets exhibited similar width of their hydrogen-rich core $\left(X_{\mathrm{H} 2} \approx 100 \%\right)$ up to about $5 D$ downstream of the nozzle exit. However, the jet under $\sim 5$ bar ambient pressure showed slightly wider cone angle from the beginning. Past $Z \approx 5 D$ downstream of the nozzle exit, the rich hydrogen core of both jets started diffusing more in the radial direction. Due to the higher momentum at the nozzle exit of the jet that was issued into the higher ambient pressure, the KelvinHelmholtz instabilities induced by the supersonic shear layer were relatively stronger, therefore, the radial expansion of this jet (and thus its cone angle) became wider. It can also be seen that, although both jets exhibited almost identical $X_{\mathrm{H} 2}$ in the vicinity of the nozzle's centreline, the jet issued into $\sim 5$ bar pressure had significantly higher values of $X_{\mathrm{H} 2}$ in the radial direction, especially for distances larger than $Y / D \approx 2$.
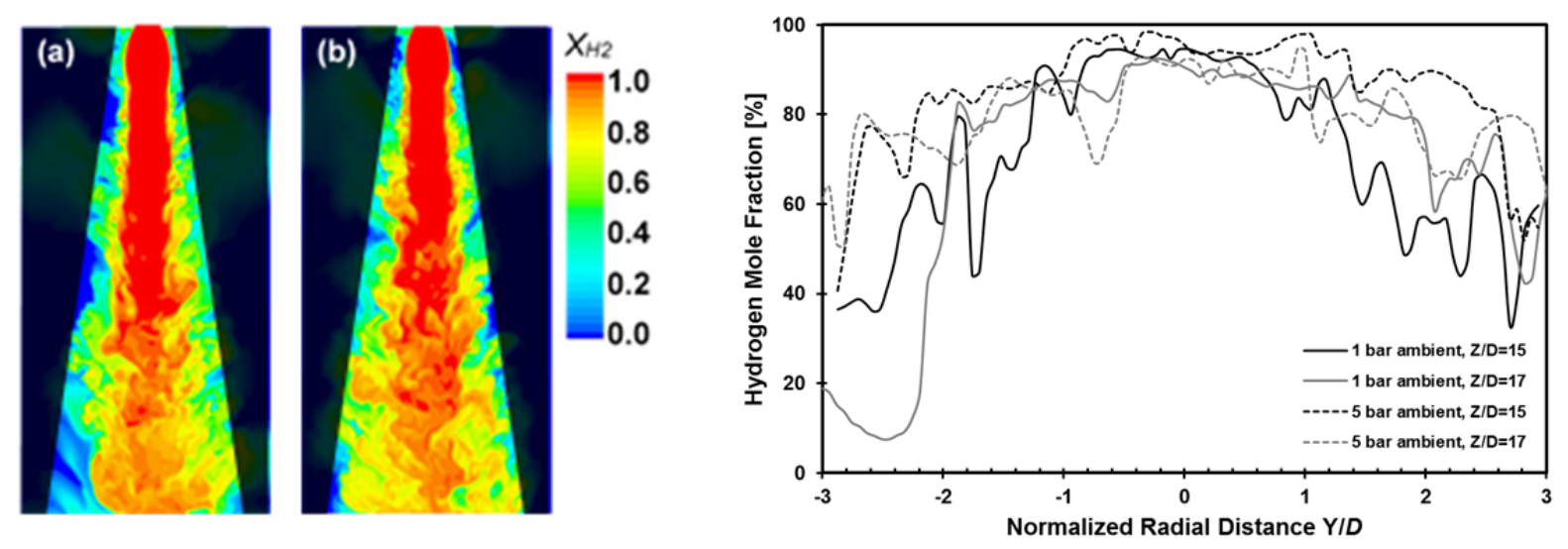

Figure 17. Left: instantaneous snapshot of $\mathrm{H}_{2}$ mole fraction at $t \approx 322 t_{0},(\mathrm{a}): \mathrm{NPR}=10$ and $P_{\infty} \approx 1 \mathrm{bar}(\mathrm{b}): \mathrm{NPR}=10$ and $P_{\infty} \approx 5$ bar. Right: $\mathrm{H}_{2}$ mole fraction probed on horizontal lines located at $\mathrm{Z}=15 \mathrm{D}$ and $17 \mathrm{D}$ downstream of the nozzle exit. 


\subsubsection{Jet Tip Penetration}

Figure 18 shows the jet tip penetration for both hydrogen and methane jets under various NPR and ambient pressure values. The injection 'delay' time, i.e. the time it took the fuel to travel inside the nozzle to the nozzle's exit, was subtracted for plotting those penetration graphs. In fact, time $t=0$ in Figure 18 is when the issuing gas is at the exit surface of the nozzle. For hydrogen and methane jets the delay time was observed to be around 4 and $6 \mu \mathrm{s}$, respectively. Methane showed considerably lower penetration when compared to hydrogen with similar NPR. Comparable result to what was observed in the current work for the tip penetration of methane and hydrogen jets at similar NPR was also reported in [54]. The shorter penetration of methane is attributed to the relatively higher speed of sound in hydrogen which resulted in higher nozzle exit velocity for the latter under-expanded jet. Based on this, since the nozzle exit velocity and maximum Mach number of the hydrogen jets with similar NPR were almost identical (see Figure 14), not much difference in the tip penetration of these jets were expected. In fact, it was found that for constant NPR lower ambient pressure resulted in slightly higher jet tip penetration. This can be attributed to the higher level of the radial momentum of the jets issued into higher ambient pressure. It was found that the greater amount of mass injected into the elevated ambient pressures got distributed radially in a stronger manner, hence a wider jet angle was produced.

As discussed in the introduction, a typical correlation for the tip penetration of a round jet is given by Equation (8). However, the values that have been reported for $C_{t}$ and $C_{f}$ relate primarily to subsonic jets [34]. Petersen and Ghandhi [28] suggested that due to the reliance of Equation (8) on the invariance of the axial momentum and moreover because of the interrupted density field (see Figure 15) in an under-expanded jet, the aforementioned equation may fail within short distances near the nozzle exit of under-expanded jets. It is still fairly hard to study precisely the initial transient behaviour of under-expanded jets issuing from a real injector for automotive applications, e.g. by means of schlieren imaging as conducted by [28]. However, as shown in the LES studies of [13-14, 42], a correlation derived from Equation (8) can be used to scale the jet tip penetration for various under-expanded jets. The current work suggests a new scaling correlation for the tip penetration of under-expanded jets based on Equation (8) as:

$$
\mathrm{Z}_{t i \mathrm{p}}=C_{A}\left(\frac{\rho_{1}}{\rho_{\infty}}\right)^{0.25}\left(U_{1} D t\right)^{0.5}
$$

where $C_{A}$ is a new parameter. The left graph of Figure 19 shows that a scaled correlation of the form $\left(\rho_{1} / \rho_{\infty}\right)^{0.25}\left(U_{1} D t\right)^{0.5}$ produces penetration curves for hydrogen and methane that have collapsed onto an almost single trend line. However, this is not telling the story of the simulated penetration curves of Figure 18. Therefore, a graph of $C_{A}$ versus time can be obtained from Figure 18 to correct the left graph of Figure 19. This is shown in the right graph of Figure 19. $C_{A}$ is a combination of $C_{t}$ and $C_{f}$ of equation (18); $C_{t}$ is related to the diffusivity and kinematic momentum of the jet whilst $C_{f}$ is associated with the jet's centreline velocity. $C_{t}$ and $C_{f}$ are significantly different for the hydrogen and methane jets which results in larger values of $C_{A}$ for hydrogen. However, if one used the constants $C_{t}$ and $C_{f}$ of [34], a value of $C_{A}$ of $\sim 2.4$ would be obtained which broadly lies on the horizon of the $C_{A}$ curves shown in Figure 19. By fitting second order polynomial 
trend lines for curves of $C_{A}$ in Figure 19 it is possible to derive correlations defining variation of $C_{A}$ versus time in form of $C_{A}=\mathrm{A} t^{2}+\mathrm{Bt}+\mathrm{C}$. Based on Figure 19 for the methane jet values of $-13.27,11.09$ and 1.12 were calculated for the coefficient $\mathrm{A}, \mathrm{B}$ and $\mathrm{C}$, respectively. Due to the very similar values of $C_{A}$ for all the hydrogen jets, a single second order polynomial was fitted on a curve representing the average of the $C_{A}$ curve of the hydrogen jets. In this case, the values of A, B and C were calculated to be $-34.65,18.71$ and 1.33 , respectively. It should be noted that the aforementioned $C_{\mathrm{A}}$ correlation and its associated $\mathrm{A}, \mathrm{B}$ and $\mathrm{C}$ coefficient values are valid for $t \geq 4 \mu \mathrm{s}$ and $t \geq 1 \mu \mathrm{s}$ for methane and hydrogen jets, respectively.

As it has been discussed in [14], due to relatively longer initial transient process and greater radial penetration of hydrogen jets with $\mathrm{NPR}=30$ and 70 compared to those with $\mathrm{NPR}=8.5$ and 10 , the scaled tip penetration did not collapse onto the curve for NPR $=8.5$ and 10 . Therefore, $C_{A}$ may need further adjustment for under-expanded jets of a specific gas with noticeably different NPR levels; this is being investigated by the current authors and will be reported in a future publication.
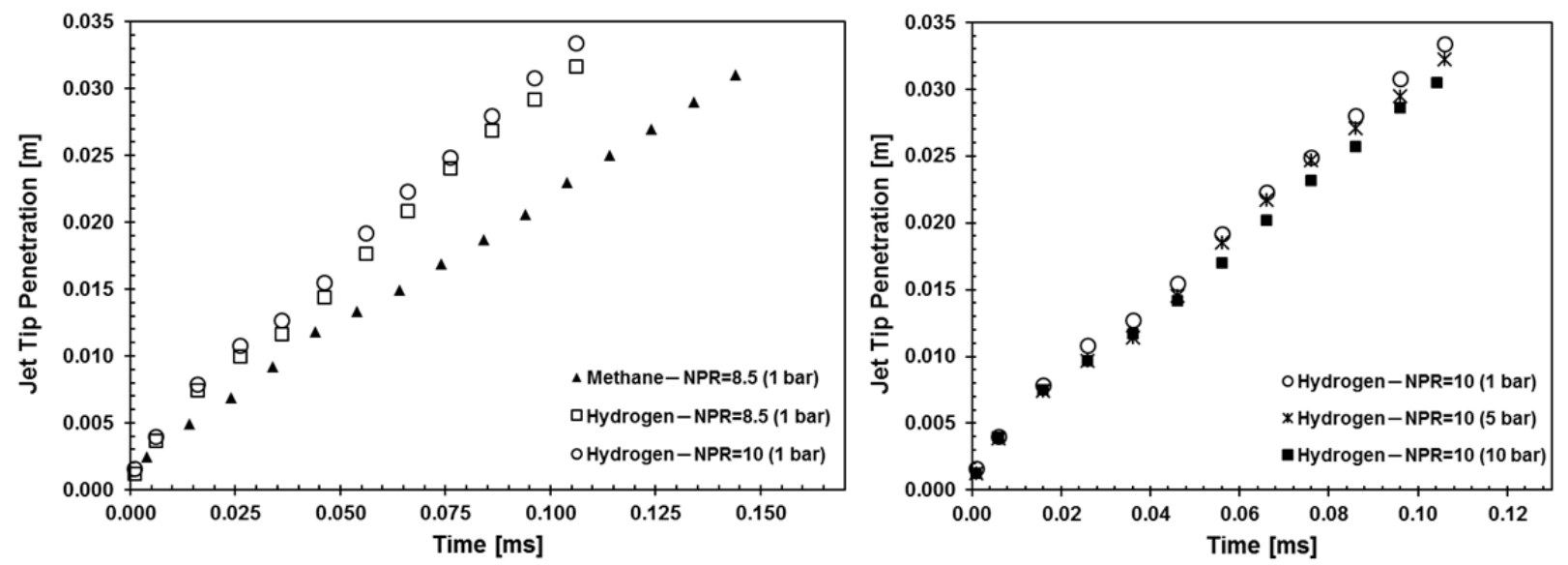

Figure 18. Jet tip penetration of under-expanded methane and hydrogen jets under various NPR and ambient pressures.
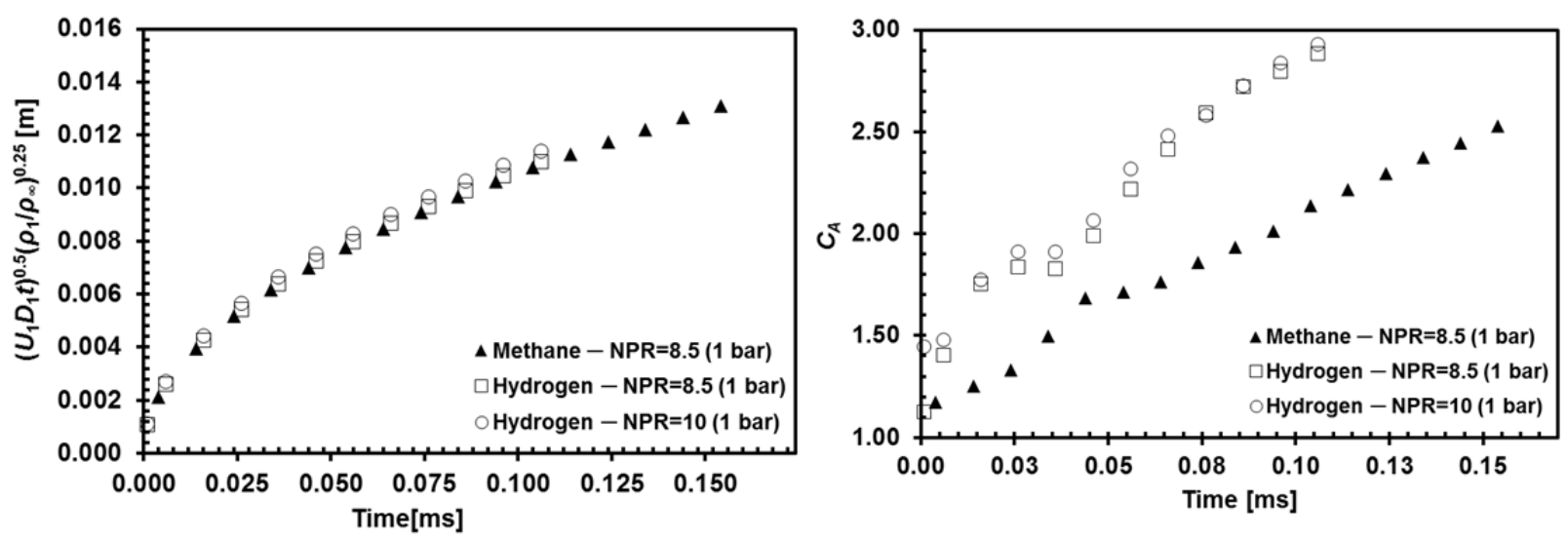

Figure 19. Left: Jet tip penetration in relation to Equation (11). Right: Variation of parameter $C_{A}$ of Equation (11) against time. 


\section{CONCLUSIONS}

The current study used large eddy simulation in order to extend the previous work of the current authors [14] by looking into the effect of elevating ambient pressure (to levels relevant to DI engines) on the key sonic and mixing characteristics of hydrogen jets. Additionally, the transient formation of the near-nozzle shock structure and initial vortex ring were studied for hydrogen and methane. The key conclusions of the present study can be summarised as follows:

- At constant NPR, higher ambient pressure resulted in faster formation of the final Mach reflection (Mach disk) and shorter Mach disk settlement time. This was attributed to the relatively faster transient evolution of the in-nozzle flow and hence fairly faster transformation from subsonic to sonic/supersonic nozzle exit for the jets at elevated ambient pressure.

- The tip vortices of the jet issued into elevated pressure conveyed more mass and transported relatively greater level of radial momentum, resulting in fairly larger radial penetration and jet cone angle.

- Significant difference in the evolution of the tip vortices was observed between hydrogen and methane at constant NPR. This was attributed to their majorly different speeds of sound and hence nozzle exit velocities.

- The initial transient tip vortices (vortex ring) of hydrogen jets may contribute significantly to the flow instabilities at the boundary of the intercepting shock and promote hydrogen-air mixing upstream of the Mach reflection (unlike for the methane jet).

- The origin of the outer shear layer for hydrogen was located about half nozzle diameter downstream of the nozzle exit very close to the centroid of the initial tip vortices. For methane, the outer shear layer originated after the Mach disk and was dominated by the Mach reflection.

- For a particular gas the transient evolution of the tip vortices (vortex ring) is mostly affected by NPR rather than the level of incoming momentum (i.e. injection pressure).

- For methane, due to the higher gradient of the transverse velocity component just before the Mach reflection, a relatively more concave Mach disk was formed than for hydrogen.

- The current LES study, in agreement with previous experimental observations, did not capture any kind of flow recirculation just downstream of the Mach disk. To the best of the authors' knowledge, this is the first time that such an observation has been made by numerical simulation of the Navier-Stokes equations.

- The near-nozzle shock structure was only affected by NPR. At constant NPR, identical Mach disk dimensions, reflected shock angles, shock cell spacing and shear layer thickness were observed for jets issued into different ambient pressures. 
- For constant NPR lower ambient pressure resulted in slightly higher jet tip penetration. This was attributed to the higher level of the radial momentum in jets issued into elevated ambient pressures.

- By calculating the probability of a density-weighted function based on the mass fraction of hydrogen it was found that a locally richer mixture existed for jets with higher NPR or with higher ambient pressure at constant NPR.

- Two correlations were proposed for the core shock cell spacing and the jet tip penetration of highly under-expanded jets.

\section{ACKNOWLEDGEMENTS}

The authors acknowledge the use of University College London's Legion High Performance Computing Facility (Legion@UCL) and associated support services, in the completion of this work. The authors would also like to thank all members of the UCL Internal Combustion Engines and Fuel Systems Group for their assistance and many valuable discussions. 


\section{APPENDIX}
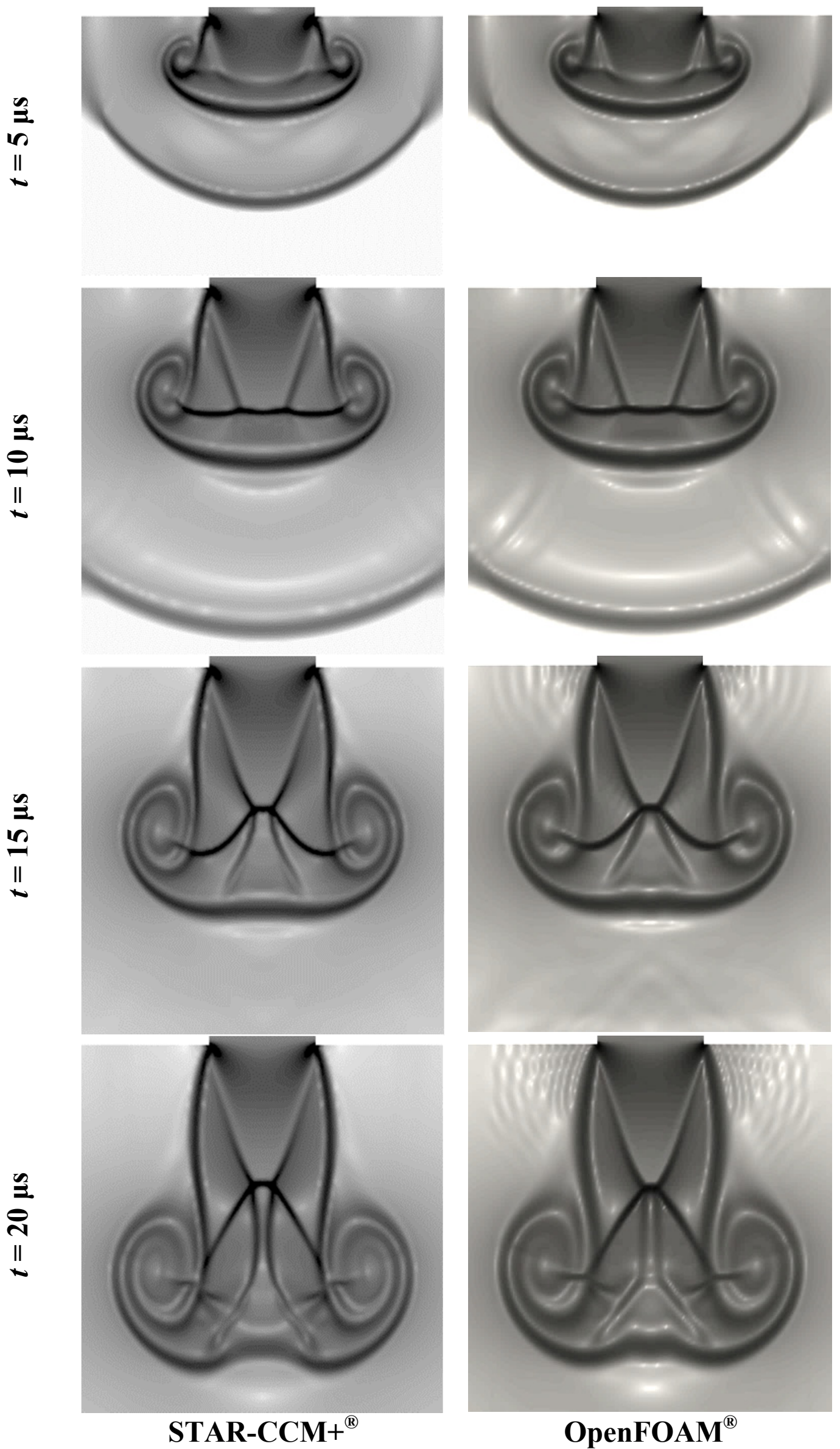

Figure A.1 Transient evolution of the near-nozzle shock structure in an under-expanded nitrogen jet. 

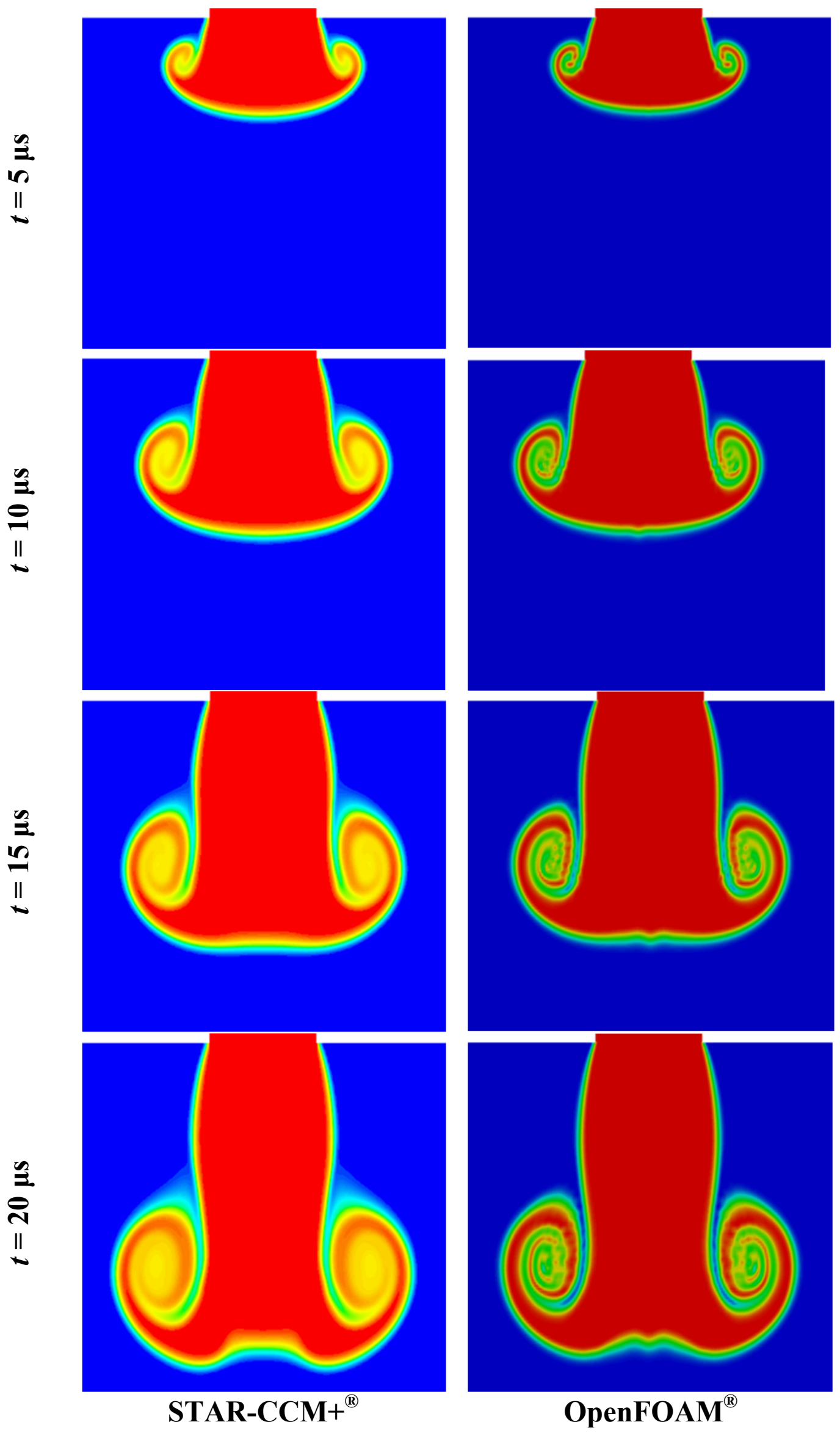

Figure A.2 Transient evolution of the initial tip vortices (vortex ring) in an under-expanded nitrogen jet (based on the scalar mass fraction). 


\section{REFERENCES}

[1] Verhelst S, Wallner T. Hydrogen-fueled internal combustion engines. Prog Energy Combust Sci 2009; 35: 490-527.

[2] Cho HM, He B. Spark ignition natural gas engines-a review. Energy Conversion and Management 2007; 48: 608-618.

[3] Scarcelli R, Wallner T, Matthias N, Salazar V, Kaiser S. Mixture formation in direct injection hydrogen engines: cfd and optical analysis of single- and multi-hole nozzles. SAE Int J Engines 2011: 2361-2375.

[4] Hamzehloo A, Aleiferis PG. Computational study of hydrogen direct injection for internal combustion engines. 2013. SAE Technical Paper 2013-01-2524.

[5] Hamzehloo A, Aleiferis PG. Numerical modelling of mixture and combustion in DISI hydrogen engines with various injection strategies. 2014. SAE Technical Paper 2014-01-2577.

[6] Crist S, Sherman PM, Glass DR. Study of the highly underexpanded sonic jet. AIAA J 1996; 4: 68-71.

[7] Donaldson CDuP, Snedeker RS. A study of free jet impingement. part 1. mean properties of free and impinging jets. J Fluid Mech 1971; 45: 281-319.

[8] Hornung H. regular and Mach reflection of shock waves. Ann Rev Fluid Mech 1986; 18: 33-58.

[9] Inman JA, Danehy PM, Nowak RJ, Alderfer DW. Identification of instability modes of transition in underexpanded jets. $38^{\text {th }}$ Fluid Dynamics Conference and Exhibit, 2008, Seattle, Washington; USA, AIAA Paper 2008-4389.

[10] Edgington-Mitchell D, Honnery RD, Soria J. The underexpanded jet Mach disk and its associated shear layer. Phys Fluids 2014; 26: 096101.

[11] Ashkenas H, Sherman FS. The structure and utilization of supersonic free jets in low density wind tunnel. In: Advances in applied mechanics-rarefied gas dynamics. New York: Academic Press; 1965. p. 84105.

[12] Velikorodny A, Kudriakov S. Numerical study of the near-field of highly underexpanded turbulent gas jets. Int J Hydrog Energy 2012; 37: 17390-17399.

[13] Vuorinen V, Yu J, Tirunagari S, Kaario O, Larmi M, Duwig C, et al. Large-eddy simulation of highly underexpanded transient gas jets. Phys Fluids 2013; 25: 016101.

[14] Hamzehloo A, Aleiferis PG. Large eddy simulation of highly turbulent under expanded hydrogen and methane jets for gaseous-fuelled internal combustion engines. Int J Hydrog Energy 2014; 39: 21275-21296.

[15] Tam CKW. Broadband shock-associated noise of moderately imperfectly expanded supersonic jets. J Sound Vib 1990; 140: 55-71.

[16] Tam C KW. Supersonic jet noise. Ann Rev Fluid Mech 1995; 27: 17-43.

[17] Panda J. Shock oscillation in underexpanded screeching jets. J Fluid Mech 1998; 363: 173-198.

[18] Pack DC. A note on Prandtl's formula for the wave-length of a supersonic gas jet. Q. J. Mech. Appl. Math 1950; 3: 173-181.

[19] Emden R. Über die ausströmungserscheinungen permanenter gase. Annalen der Physik 1899; 305: $264-$ 289.

[20] Prandtl L. Stationary waves in a gaseous jet. Physikalische Zeitschrift 1904; 4: 599-601. 
[21] Tam CKW, Tanna HK. Shock associated noise of supersonic jets from convergent-divergent nozzles. J Sound Vib 1982; 81: 337-358.

[22] Tam CKW, Jay AJ, Seiner JM. A multiple-scales model of the shock-cell structure of imperfectly expanded supersonic jets. J Fluid Mech 1985; 153: 123-149.

[23] $\mathrm{Hu} \mathrm{TF}$, McLaughlin DK. Flow and acoustic properties of low reynolds number underexpanded supersonic jets. J Sound Vib 1990; 141: 485-505.

[24] Scroggs SD, Settles GS. An experimental study of supersonic microjets. Exp. Fluids 1996; 21: 401-409.

[25] Phalnikar KA, Kumar R, Alvi FS. Experiments on free and impinging supersonic microjets. Exp. Fluids 2008; 44: 819-830.

[26] Ouellette P. Direct injection of natural gas for diesel engine fueling. PhD Thesis. 1996. The University of British Columbia, Vancouver, Canada.

[27] Ouellette P, Hill PG. Turbulent transient gas injections. J Fluids Eng 2000; 122: 743-752.

[28] Petersen BR, Ghandhi JB. Transient high-pressure hydrogen jet measurements. 2006. SAE Technical Paper 2006-01-0652.

[29] Turner JS. The 'starting plume' in neutral surroundings. J Fluid Mech 1962; 13: 356-368.

[30] Hill PG, Ouellette P. Transient turbulent gaseous fuel jets for diesel engines. J Fluids Eng 1999; 121: 93-101.

[31] Ricou FP, Spalding DB. Measurements of entrainment by axisymmetrical turbulent jets. J Fluid Mech 1961; 11:21-32.

[32] Abraham J. Entrainment characteristics of transient gas jets. . Heat Transf. Part A: Appl 1996; 30: 347364.

[33] Schlichting H. Boundary layer theory. McGraw-Hill, New York. 1976.

[34] Song L, Abraham J. Entrainment characteristics of transient turbulent round, radial and wall-impinging jets: theoretical deductions. J Fluids Eng 2003; 125: 605-612.

[35] Dauptain A, Cuenot B, Gicquel YM. Large-eddy simulation of a stable supersonic jet impinging on flat plate. AIAA J 2010; 48: 2325-2337.

[36] White T, Milton B. Shock wave calibration of under expanded natural gas fuel jets. Shock Waves 2008; 18: 353-364.

[37] Vuorinen V, Wehrfritz A, Duwig C, Boersma BJ. Large-eddy simulation on the effect of injection pressure and density on fuel jet mixing in gas engines. Fuel 2014; 130: 241-250.

[38] Khaksarfard R, Kameshki MR, Paraschivoiu M. Numerical simulation of high pressure release and dispersion of hydrogen into air with real gas model. Shock Waves 2010; 20: 205-216.

[39] Gorlé C, Gamba M, Ham F. Investigation of an underexpanded hydrogen jet in quiescent air using numerical simulations and experiments. In: Annual research briefs. Center for Turbulence Research, Stanford University; 2010.

[40] Ruggles AJ, Ekoto IW. Ignitability and mixing of underexpanded hydrogen jets. Int J Hydrog Energy 2012; 37: 17549-17560. 
[41] Bonelli F, Viggiano A, Magi V. A numerical analysis of hydrogen underexpanded jets under real gas assumption. J Fluids Eng 2013; 135: 121101.

[42] Hamzehloo A, Aleiferis PG. Large eddy simulation of near-nozzle shock structure and mixing characteristics of hydrogen jets for direct-injection spark-ignition engines. $10^{\text {th }}$ International Conference on Heat Transfer, Fluid Mechanics and Thermodynamics (HEFAT2014), Orlando, Florida, USA, 2014.

[43] Weiss JM, Smith WA. Preconditioning applied to variable and constant density flows. AIAA J 1995; 33: 2050-2057.

[44] Weiss JM, Maruszewski JP, Smith WA. Implicit solution of preconditioned Navier-Stokes equations using algebraic multigrid. AIAA J 1999; 37: 29-36.

[45] Ferziger JH, Peric M. Computational methods for fluid dynamics. Springer. 2002.

[46] Liou MS. A sequel to AUSM, part II: AUSM +-up for all speeds. J. Comput. Phys. 2006; 214: 137170.

[47] Pope SB. Turbulent flows. Cambridge University Press. 2000.

[48] Nicoud F, Ducros F. Subgrid-scale stress modelling based on the square of the velocity gradient tensor. Flow Turbul Combust 1999; 62: 183-200.

[49] Cussler EL. Diffusion: mass transfer in fluid systems. $3^{\text {rd }}$ Edition, Cambridge University Press. 2009.

[50] Weller HG, Tabor G, Jasak H, Fureby C. A tensorial approach to computational continuum mechanics using object orientated techniques. Comp. in Phys. 1998; 12: 620-631.

[51] Hamzehloo A. Computational study of under-expanded jets, mixture formation and combustion in direct-injection spark-ignition hydrogen engines. PhD Thesis, University College London (UCL), UK, 2015.

[52] André B, Castelain T, Bailly C. Investigation of the mixing layer of underexpanded supersonic jets by particle image velocimetry. Int J Heat Fluid Flow 2014; 50: 188-200.

[53] Golub VV. Development of shock wave and vortex structures in unsteady jets. Shock Waves 1994; 3 : 279-285.

[54] Rogers T, Petersen P, Koopmans L, Lappas P, Boretti A. Structural characteristics of hydrogen and compressed natural gas fuel jets. Int J Hydrog Energy 2015; 40: 1584-1597.

[55] Cabral B, Leedom LC. Imaging vector fields using line integral convolution. Proceedings of the $20^{\text {th }}$ Annual Conference on Computer Graphics and Interactive Techniques, ACM, 1993.

[56] Smarr LL, Michael LN, Winkler KA. Shocks, interfaces and patterns in supersonic jets. Physica D: Nonlinear Phenomena 1984; 12: 83-106.

[57] Bulgakov AV, NM Bulgakova. Gas-dynamic effects of the interaction between a pulsed laser-ablation plume and the ambient gas: analogy with an underexpanded jet. J. Phys. D. Appl. Phys 1998; 31: 693-70.

[58] Saric WSM. Görtler vortices. Ann Rev Fluid Mech 1994; 26: 379-409.

[59] André B, Castelain T, Bailly C. Experimental exploration of underexpanded supersonic jets. Shock Waves 2014; 24: 21-32.

[60] Maté B, Graur IA, Elizarova T, Chirokov I, Tejeda G, Fernandez JM, Montero S. Experimental and numerical investigation of an axisymmetric supersonic jet. J Fluid Mech 2001; 426: 177-197. 
[61] Gribben BJ, Badcock KJ, Richards BE. Numerical study of shock-reflection hysteresis in an underexpanded jet. AIAA J 2000; 38: 275-283.

[62] Skovorodko PA, Levin DA, Wysong IJ, Garcia AL. About the nature of the recirculation zone behind a mach disk in an underexpanded jet. AIP Conference Proceedings-American Institute of Physics 2011; 1333: 601-606.

[63] Frey M, Hagemann G. Restricted shock separation in rocket nozzles. J Prop Power, 2000; 16: 478-484. 